\title{
1 Photoinduced Isomerization Sampling of Retinal in 2 Bacteriorhodopsin
}

3

4 Zhong Ren

5

6 Department of Chemistry, University of Illinois at Chicago, Chicago, IL 60607, USA

7 Renz Research, Inc., Westmont, IL 60559, USA

8

9 zren@uic.edu

10 ORCID 0000-0001-7098-3127

\section{Keywords}

13 charge separation, proton pump, serial crystallography, singular value decomposition,

14 X-ray free electron laser

\section{Abstract}

Photoisomerization of retinoids inside a confined protein pocket represents a critical chemical event in many important biological processes from animal vision, non-visual light effects, to bacterial light sensing and harvesting. Light driven proton pumping in bacteriorhodopsin entails exquisite electronic and conformational reconfigurations during its photocycle. However, it has been a major challenge to delineate transient molecular events preceding and following the photoisomerization of the retinal from noisy electron density maps when varying populations of intermediates coexist and evolve as a function of time. Here I report several distinct early photoproducts deconvoluted from the recently observed mixtures in time-resolved serial crystallography. This deconvolution substantially improves the quality of the electron density maps hence demonstrates that the all-trans retinal undergoes extensive isomerization sampling before it proceeds to the productive 13-cis configuration. Upon light absorption, the chromophore attempts to perform trans-to-cis isomerization at every double bond coupled with the stalled anti-to-syn rotations at multiple single bonds along its polyene chain. Such isomerization sampling pushes all seven transmembrane helices to bend outward, resulting in a transient expansion of the retinal binding pocket, and later, a contraction due to recoiling. These ultrafast 


\section{Ren: Isomerization sampling}

responses observed at the atomic resolution support that the productive photoreaction in bacteriorhodopsin is initiated by light-induced charge separation in the prosthetic chromophore yet governed by stereoselectivity of its protein pocket. The method of a numerical resolution of concurrent events from mixed observations is also generally applicable.

\section{Introduction}

Bacteriorhodopsin (bR) pumps protons outward from the cytoplasm $(\mathrm{CP})$ against the concentration gradient via photoisomerization of its retinal chromophore. The trimeric $\mathrm{bR}$ on the native purple membrane shares the seven transmembrane helical fold and the same prosthetic group (Fig. S1) with large families of microbial and animal rhodopsins (Ernst et al., 2014; Kandori, 2015). An all-trans retinal in the resting state is covalently linked to Lys216 of helix $\mathrm{G}$ through a Schiff base (SB), of which the double bond $\mathrm{C}_{15}=\mathrm{N}_{\zeta}$ is also in trans. Upon absorption of a visible photon, the all-trans retinal in $b R$ isomerizes efficiently and selectively to adopt the 13-cis configuration (Govindjee et al., 1990). In contrast, an all-trans free retinal in organic solvents could isomerize about various double bonds, but with poor quantum yields (Freedman and Becker, 1986; Koyama et al., 1991).

A broad consensus is that the isomerization event takes place around 450-500 fs during the transition from a blue-shifted species I to form a red-shifted intermediate J (Herbst, 2002; Mathies et al., 1988). Various molecular events prior to the isomerization have also been detected. Vibrational spectroscopy showed a variety of possible motions, such as torsions about $\mathrm{C}_{13}=\mathrm{C}_{14}$ and $\mathrm{C}_{15}=\mathrm{N}_{\xi}$, H-out-of-plane wagging at $\mathrm{C}_{14}$, and even protein responses (Diller et al., 1995; Kobayashi et al., 2001). Nevertheless, the species I or a collection of species detected before $30 \mathrm{fs}$ remain in a good trans configuration about $\mathrm{C}_{13}=\mathrm{C}_{14}$ instead of a near $90^{\circ}$ configuration (Zhong et al., 1996). Recently, deep-UV stimulated Raman spectroscopy revealed strong signals of Trp and Tyr motions in the protein throughout the I and J intermediates (Tahara et al., 2019). Despite extensive studies, fundamental questions on the photoisomerization of retinal remain unanswered at the atomic resolution. What is the quantum mechanical force that causes the all-trans retinal to isomerize specifically to 13-cis after absorbing a photon? Why not isomerize elsewhere in bR? How is the quantum yield of this specific isomerization enhanced by the protein compared to those of free retinal in solution? 


\section{Ren: Isomerization sampling}

68 Does any isomerization sampling occur? This work addresses these questions by 69 solving a series of structures of the early intermediates based on the electron density 70 maps unscrambled from the published serial crystallography datasets using singular 71 value decomposition (SVD). These structures of "pure" photoproducts at atomic

72 resolution reveal widespread conformational changes in all seven helices prior to the 73 all-trans to 13-cis isomerization and after its completion, suggesting that isomerization 74 sampling takes place in $\mathrm{bR}$, where rapid photoisomerizations and single bond rotations 75 are attempted everywhere along the polyene chain of the retinal before the only 76 successful one flips the SB at $\sim 500 \mathrm{fs}$.

Several international consortiums carried out large operations of serial crystallography at free electron lasers (XFELs). It is now possible to capture transient structural species at room temperature in the bR photocycle as short-lived as $\mathrm{fs}$ (Brändén and Neutze, 2021). Compared to cryo-trapping, authentic structural signals from these XFEL data are expected to be greater in both amplitude and scope. However, the signals reported so far do not appear to surpass those obtained by cryotrapping methods, suggesting much needed improvements in experimental protocols and data analysis methods. Two major sources of data are used in this study (Table S1). Nogly et al. captured retinal isomerization to 13-cis by the time of 10 ps and attributed the specificity to the H-bond breaking between the SB and a water (Nogly et al., 2018). Kovacs et al. contributed datasets at many short time delays (Kovacs et al., 2019). Those sub-ps datasets demonstrate oscillatory signals at frequencies around $100 \mathrm{~cm}^{-1}$. The essence of this work is a numerical resolution of structural heterogeneity, a common difficulty often encountered in cryo trapping and time-resolved serial crystallography. To what extend a specific structural species can be enriched in crystals depends on the reaction kinetics governed by many experimental parameters including but not limited to the fluence, wavelength, and temperature of the light illumination. While it is possible to reach higher fractional concentrations at specific time points for more stable species such as $\mathrm{K}$ or $\mathrm{M}$ due to the ratio between the rates going into and exiting from that species, transient species such as I and J are often poorly populated. If such structural heterogeneity is not resolved, it is very difficult, if not impossible, to interpret the electron density maps and to refine the intermediate structures (Ren et al., 2013). An assumption in nearly all previous studies has been that each dataset, at a cryo temperature or at a time delay, is derived from a mixture of a single photoinduced 


\section{Ren: Isomerization sampling}

102

103

104

105

106

107

108

109

110

111

112

113

114

115

116

117

118

119

120

121

122

123

124

125

126

127

128

129

130

131

132

133

134

135

species and the ground state. Therefore, the difference map reveals a pure intermediate structure. This assumption is far from the reality thus often leads to misinterpretation of the observed electron density maps. This work is yet another case study to demonstrate the application of our analytical protocol based on SVD (Methods) that makes no assumption on how many excited intermediates that contribute to the captured signals at each time point (Ren, 2019; Ren et al., 2013; Yang et al., 2011). More importantly, this work showcases that our resolution of structural heterogeneity enables new mechanistic insights into the highly dynamic chemical or biochemical processes.

\section{Results and Discussion}

A total of 24 datasets and 18 time points up to 10 ps are analyzed in this study (Table S1). Difference Fourier maps at different time points and with respect to their corresponding dark datasets are calculated according to the protocols previously described (Methods). A collection of 126 difference maps at short delays $\leq 10$ ps are subjected to singular value decomposition (SVD; Methods) followed by a numerical deconvolution using the previously established Ren rotation in a multi-dimensional Euclidean space (Ren, 2016, 2019). Such resolution of electron density changes from mixed photoexcited species in the time-resolved datasets results in four distinct intermediate structures in the early photocycle, which are then refined against the reconstituted structure factor amplitudes (Table S2; Methods).

\section{Low frequency oscillations observed upon photoexcitation}

Ten out of 17 major components derived from the sub-ps delays of Kovacs et al. (Fig. S2) describe five two-dimensional oscillatory behaviors at frequencies ranging from 60 to $400 \mathrm{~cm}^{-1}$ (Fig. S3). Compared to a bond stretching frequency commonly observed in vibrational spectroscopy, these oscillations are at much lower frequencies. The lowest frequency is $61 \pm 2 \mathrm{~cm}^{-1}$, that is, a period of $550 \pm 20 \mathrm{fs}$ (Fig. S3a), which matches exactly the oscillation detected in transient absorption changes in visual rhodopsin (Wang et al., 1994). Although these ten components follow the oscillatory time dependencies, they do not show any association with the chromophore or the secondary structure of the protein (Fig. S4). Similar oscillatory components were also present in the XFEL datasets of $\mathrm{MbCO}$ (Ren, 2019). Therefore, the same conclusion stands that these low frequency vibrations induced by short laser pulses often detected by ultrafast spectroscopy are the intrinsic property of a solvated protein molecule, here specifically bacteriorhodopsin 


\section{Ren: Isomerization sampling}

136

137

138

139

140

141

142

143

144

145

146

147

148

149

150

151

152

153

154

155

156

157

158

159

160

161

162

163

164

165

166

167

168

(bR) (Johnson et al., 2014; Liebel et al., 2014). Interestingly, the isomerization sampling and productive photoisomerization observed in this study occur within the first oscillatory period at the lowest frequency. While such coincidence begs the question whether the protein oscillation is required for isomerization (see below), direct evidence is lacking in these XFEL data to support any functional relevance of these oscillatory signals.

\section{Intermediates $I^{\prime}, I$, and expansion of retinal binding pocket}

In contrast to the oscillating signals, three components $\boldsymbol{U}_{10}, \boldsymbol{U}_{14}$, and $\boldsymbol{U}_{17}$ reveal strong light-induced structural signals in terms of both extensiveness and quality (Figs. 1ab and S5). These signals originate exclusively from a few time points of Nogly et al., too

few to fit the time dependency with exponentials. Instead, a spline fitting through these time points gives rise to the estimated coefficients $c_{10}, c_{14}$, and $c_{17}$ in the linear combination of ${ }_{110} \boldsymbol{U}_{10}+{ }_{C_{14}} \boldsymbol{U}_{14}+{ }_{c_{17}} \boldsymbol{U}_{17}$ for reconstructing the electron density maps of the states I, J, and their respective precursors I', J' (Fig. 2a). A reconstituted difference map of $\mathrm{I}^{\prime}-\mathrm{bR}$ (Fig. 1c) is located on the spline trajectory from the origin, that is, $b R$ at the time point of $0-$, to the first time point of $49-406 \mathrm{fs}$ (PDB entry 6g7i). This state is denoted I' as a precursor leading to the I state judged by the time point at $~ 30 \mathrm{fs}$. However, this is not to say that a single species I' exists around $30 \mathrm{fs}$. Quite the opposite, the population of the time-independent conformational species I' rises and falls and peaks around $30 \mathrm{fs}$, while many other species during isomerization sampling coexist with I' at the same time (see below). The reconstituted difference map is used to calculate a set of structure factor amplitudes that would produce this difference map of $\mathrm{I}^{\prime}-\mathrm{bR}$ (Methods). And the structure of $\mathrm{I}^{\prime}$ is refined against this reconstituted dataset (beige; Figs. 1cd and S6). The same protocol is used to refine the structure of I state (purple; Fig. S7) with a reconstituted difference map I - bR (Figs. 1a, 2ab, 3a, and S5). This SVD-dependent refinement strategy extends the commonly used method based on an extrapolated map to another level. This newly developed method is able to refine a structure against any linear combination of signal components while eliminating noise and systematic error components, and components identified as other intermediate species mixed in the data. Therefore, this method enables the refinement of an unscrambled, hence pure, structural species (Methods). 


\section{Ren: Isomerization sampling}

169

170

171

172

173

174

175

176

177

178

179

180

181

182

183

184

185

186

187

188

189

190

191

192

193

194

195

196

197

198

199

200

201

202

The all-trans retinal chromophore in the ground state of $\mathrm{bR}$ is largely flat except the last atom $\mathrm{C}_{15}$ (Fig. 2c $2^{\text {nd }}$ panel). In contrast, the side chain of Lys216 is highly twisted forming two near- $90^{\circ}$ single bonds (Fig. $2 \mathrm{c} 4^{\text {th }}$ panel), which results in a corner at $\mathrm{C}_{\varepsilon}$ that deviates dramatically from the plane of the all-trans retinal (Fig. 2c $2^{\text {nd }}$ panel). The refined geometry of the retinal in I' retains a near perfect all-trans configuration, including the Schiff base (SB) double bond $\mathrm{C}_{15}=\mathrm{N}_{\zeta}$, while various single bonds along the polyene chain deviate from the standard anti conformation significantly (Fig. 2c $4^{\text {th }}$ panel). The torsional deviations from anti are in a descending order from the $\beta$-ionone ring to the SB. These torsional changes result in an S-shaped retinal shortened by $\sim 4 \%$ (Fig. 2c $3^{\text {rd }}$ panel). The distal segment $\mathrm{C}_{6}-\mathrm{C}_{12}$ moves inboard up to $0.9 \AA$ and the proximal segment $\mathrm{C}_{13}-\mathrm{C}_{\varepsilon}$, including the $\mathrm{SB}$, moves outboard up to $1.6 \AA$ (Fig. 2c $1^{\text {st }}$ and $2^{\text {nd }}$ panels; see Fig. S1 for orientations in $b R$ ). This creased retinal observed here at around $30 \mathrm{fs}$ (Fig. 1d) is attributed to the direct consequence of a compression under an attraction force between the $\beta$-ionone ring and the SB (see below).

The refined structure of the I state (Fig. S7) shows that the retinal remains in near perfect all-trans, including the SB, and as creased as its precursor I' (Fig. 3c). The torsional deviations from anti single bonds become even more severe compared to the $\mathrm{I}^{\prime}$ state and remain in a descending order from the $\beta$-ionone ring to the SB (Fig. $2 \mathrm{c} 4^{\text {th }}$ panel). The major difference from its precursor is that the single bond $\mathrm{N}_{\zeta}-\mathrm{C}_{\varepsilon}$ now adopts a perfect syn conformation (Figs. 2c $4^{\text {th }}$ panel and 3c), and the anchor Lys216 has largely returned to its resting conformation. Such a lack of substantial change between the ground state and the intermediate I was previously noted by a comparison of a chemically locked $\mathrm{C}_{13}=\mathrm{C}_{14}$ with the native retinal (Zhong et al., 1996).

Remarkably, the major component $\boldsymbol{U}_{10}$ reconstituted into the difference map of I - bR contains widespread signal associated with all seven helices (Fig. 2b). The reconstituted map clearly shows collective outward motions from the center (Fig. 3a) suggesting an expansion of the retinal binding pocket at hundreds of $\mathrm{fs}$, which is confirmed by the refined structure of the I state (Fig. $3 \mathrm{~d}$ top panel). For example, the distance between the $\mathrm{C}_{\alpha}$ atoms increases by $0.8 \AA$ between Arg82 and Phe208 and by $0.7 \AA$ between Tyr83 and Trp182. It is noteworthy that similar protein signals are present in the raw difference map calculated from the time point of 457-646 fs from Nogly et al. (6g7j) prior to an SVD analysis (Fig. S8). 


\section{Ren: Isomerization sampling}

Transient bleaching at near UV of 265-280 nm was observed before $200 \mathrm{fs}$ and attributed to structural changes in the retinal skeleton and the surrounding Trp residues (Schenkl et al., 2005). Recent deep-UV stimulated Raman spectroscopy also demonstrated that motions of Trp and Tyr residues start to emerge at $200 \mathrm{fs}$ and remain steady until the isomerization is over at 30 ps (Tahara et al., 2019). Here the refined structure of the I state with displaced helices and an expanded retinal binding pocket offers an explanation for the stimulated Raman gain change at hundreds of fs. However, it is unclear why and how such extensive protein responses take place even before the retinal isomerization. According to the broadly accepted concept of proteinquake, initial motions are generated at the epicenter where the chromophore absorbs a photon and then propagated throughout the protein matrix (Ansari et al., 1985). It is plausible that these ultrafast protein responses are the direct consequence of isomerization sampling in a confined protein pocket. It was observed in organic solvents using high-pressure liquid chromatography (HPLC) that all-trans retinal could isomerize at various double bonds along the polyene chain to adopt 9-, 11-, and 13-cis configurations, but with rather poor quantum yields (Freedman and Becker, 1986; Koyama et al., 1991). This intrinsic property of the all-trans retinal would behave the same even when it is incorporated in the protein except that the protein matrix herds the chromophores on the right track of the productive photocycle and keeps the concentrations of the attempted byproducts low. These byproduct conformations of the retinal during isomerization sampling are too numerous and too minor to be observed experimentally. Nevertheless, they cause a common effect, an expansion of its binding pocket, since the all-trans retinal in the resting state is tightly boxed by massive side chains all around (Fig. 3e). Any attempt to isomerize would push against this box one way or another. For instance, triple attempts to isomerize simultaneously at 11, 13, and 15 positions were suggested by a quantum mechanics/molecular mechanics simulation (Altoè et al., 2010). When the retinal binding pocket is altered in mutants, the quantum yield of each isomerization byproduct is expected to increase resulting in an impaired productive pathway (see below). 


\section{Ren: Isomerization sampling}

237

238

239

240

241

242

243

244

245

246

247

248

249

250

251

252

253

254

255

256

257

258

259

260

261

262

263

264

265

266

267

268

269

270

restoration of the normal retinal binding pocket in $\mathrm{J}^{\prime}$ from an expanded one in the I state followed by a contraction in J (Fig. 3d bottom panel). Two time-independent structures of J' (green; Fig. S9) and J (gray; Fig. S10) are refined based on the respective reconstituted difference maps with the same protocol (Methods). Their populations peak at the approximate time of $\sim 700 \mathrm{fs}$ and $\sim 20 \mathrm{ps}$, respectively. The observed contraction of the retinal binding pocket is likely due to an elastic recoiling of the seven helical bundle following its transient expansion caused by the isomerization sampling.

The creased retinal persists in both the $\mathrm{J}^{\prime}$ and J structures (Fig. 2c ${ }^{\text {nd }}$ panel and Fig. 3c). The difference map of J' - bR clearly shows the 13-cis configuration (Fig. 3b). Indeed, near perfect 13-cis is successfully refined in both structures (Fig. 2c $4^{\text {th }}$ panel). While the SB double bond $\mathrm{C}_{15}=\mathrm{N}_{\zeta}$ is momentarily distorted from the trans configuration in $\mathrm{J}^{\prime}$ with a torsion angle of $133^{\circ}$, a perfect trans configuration at $\mathrm{C}_{15}=\mathrm{N}_{\zeta}$ is promptly restored in J (Fig. 2c $4^{\text {th }}$ panel). The refined structures of this series of early intermediates show that the $\mathrm{SB} \mathrm{N}_{\zeta}$ is rotating clockwise in the entire process of the isomerization of $\mathrm{I}^{\prime} \rightarrow \mathrm{I} \rightarrow \mathrm{J}^{\prime} \rightarrow \mathrm{J}$, if the retinal is viewed from the proximal to distal direction (Fig. 2c). It seems that the isomerization starts in an expanded retinal binding pocket and finishes in a tighter one. Whether the pocket expansion and contraction are required for the productive isomerization and what role the low frequency oscillations play in isomerization will need more time points at short delays to further isolate the molecular events temporally.

\section{Coulomb attraction as driving force of isomerization sampling}

The fundamental questions remain: What is the driving force that causes the all-trans retinal to isomerize after a photon absorption, at several double bonds if not restrained but exclusively at $\mathrm{C}_{13}=\mathrm{C}_{14}$ in $\mathrm{bR}$ ? How does the protein environment enhance the quantum yield of the isomerization to 13-cis? Here I hypothesize that a Coulomb attraction between the $\beta$-ionone ring and the SB at the Frank-Condon point, $0+$ time point, provides the initial driving force upon a photon absorption. The electric field spectral measurements (Mathies and Stryer, 1976) and the quantum mechanics simulation (Nogly et al., 2018) suggested that a charge separation occurs along the polyene chain at the excited state of bR. Such a dipole moment was also detected through a transient bleaching signal at near UV region (Schenkl et al., 2005). It can be shown that a plausible charge separation of \pm 0 .1e between the $\beta$-ionone ring and the SB 


\section{Ren: Isomerization sampling}

271 would cause an attraction force $>1 \mathrm{pN}$. If calibrated with the measured range of dipole

272 moment of 10-16 D (Mathies and Stryer, 1976), the charge separation could reach the

273 level of $\pm 0.16 \mathrm{e}$ to $\pm 0.26 \mathrm{e}$, giving rise to an attraction force of $3.5-9 \mathrm{pN}$ between the $\beta$ -

274 ionone ring and the SB. This attraction force is evidently sufficient to crease the flat all-

275 trans retinal into an S-shape and to compress it slightly within tens of fs as observed in

$276 \mathrm{I}^{\prime}$ and I states (Figs. 1d, 2c $2^{\text {nd }}$ and $3^{\text {rd }}$ panels, and 3c). In the meanwhile, this very

277 attraction force also triggers simultaneous attempts of double bond isomerizations and

278 single bond rotations along the polyene chain that cause the expansion of the retinal

279 binding pocket as observed at hundreds of fs. Following the only successful

280 isomerization at $C_{13}=C_{14}$, the chromophore segment from $C_{15}$ to $C_{\delta}$ is attracted to the $\beta$ -

281 ionone ring; and these two parts become significantly closer (Fig. 2c $3^{\text {rd }}$ panel). None of

282 the single bond rotations can complete under the restraints of the protein. Especially,

283 the segment closer to the midpoint of the retinal is more confined due to the steric

284 hinderance of Thr90 and Tyr185 from the inboard and outboard sides, respectively (Fig.

$2853 e)$. Therefore, the single bonds deviate from anti less and less towards the midpoint

286 (Fig. 2c $4^{\text {th }}$ panel). The effect of charge separation seems eased gradually as the reaction

287 proceeds beyond the $\mathrm{J}$ state as indicated by the slow restoration of the anti conformation

288 (Fig. 2c $4^{\text {th }}$ panel).

Apparently, the same charge separation and the attraction force upon photon absorption also take place in a solution sample of free retinal. Compared to the retinal embedded in protein, photoisomerization in solution is nonspecific, resulting in a range of byproducts, since an isomerization at any position would bring the SB significantly closer to the $\beta$-ionone ring. It is understandable that each of the byproducts could only achieve a poor quantum yield (Freedman and Becker, 1986; Koyama et al., 1991) as rotations at multiple single bonds driven by the same attraction force and achieving a similar folding of the polyene chain would further sidetrack the double bond isomerizations thus diminishing their quantum yields. However, these byproducts due to single bond rotations are short-lived beyond detection by HPLC as they spontaneously revert back in solution. The protein environment in bR plays a major role in enhancing the quantum yield of the isomerization to 13-cis by shutting down all

302 other reaction pathways triggered by the charge separation. This is further elucidated 303 by the mutant functions below. 


\section{Ren: Isomerization sampling}

305

306

307

308

309

310

311

312

313

314

315

316

317

318

319

320

321

322

323

324

325

326

327

328

329

330

331

332

333

334

335

336

337

338

\section{Isomerization byproducts permitted by mutant protein environments}

The structure of a double mutant T90A/D115A (3cod) showed little difference from the wildtype (Joh et al., 2008) while the single mutants T90V and T90A retain $<70 \%$ and < $20 \%$ of the proton pumping activity, respectively (Marti et al., 1991; Perálvarez et al., 2001). These observations illustrate that some nonproductive pathways of the isomerization sampling succeed more in the altered retinal binding pocket. In the wildtype structure, Thr90 in helix $C$ points towards the $C_{11}=C_{12}-C_{13}-C_{20}$ segment of the retinal from the inboard with its $C_{\gamma}$ atom $3.7 \AA$ from the retinal plane. Given the van der Waals radius $r_{c}$ of $1.7 \AA$, only $0.3 \AA$ is spared for the $\mathrm{H}$ atoms of the $\mathrm{C}_{\gamma}$ methyl group thereby effectively shutting down the nonproductive pathways of the isomerization sampling. Any motion of the retinal would have to push helix $\mathrm{C}$ toward inboard causing an expansion of its binding pocket. Missing this close contact in T90A increases the room to $1.9 \AA$ for isomerization byproducts, which would greatly reduce the quantum yield of the 13 -cis productive isomerization thus retain $<20 \%$ of the activity.

In addition to 13-cis, the retinal in the light adapted T90V mutant showed 9- and 11cis configurations at the occupancies of $3 \%$ and $18 \%$, respectively, while these configurations were not detected in light adapted wildtype (Marti et al., 1991). Then why would a $\mathrm{Val}$ residue at this position with an equivalent $\mathrm{C}_{\gamma}$ atom permit the formation of some isomerization byproducts? In wildtype bR, the side chain of Thr90 engages two strong H-bonds Trp86O-Thr90O ${ }_{\gamma}-\mathrm{D} 115 \mathrm{O}_{\delta}$ so that its $\mathrm{C}_{\gamma}$ methyl group is aligned toward the retinal. Without these H-bonds in T90V, the isopropyl group of Val90 is free to adopt other rotameric positions so that neither of the $C_{\gamma}$ methyl groups has to point directly to the retinal, which increases the available room for the formation of some isomerization byproducts. Compared to the light adapted state, these isomerization byproducts could reach even higher percentages during active photocycles thus reduce the proton pumping activity below $70 \%$.

From the outboard, the side chain of Tyr185 in helix $\mathrm{F}$ is nearly parallel to the retinal plane with a distance of $3.5 \AA$. This close contact of a flat area from $\mathrm{C}_{8}$ to $\mathrm{C}_{14}$ of the retinal prevents any significant motion of the retinal toward the outboard. Even slight motions would push helix F away as observed here in the expansion of the retinal binding pocket. The mutant Y185F largely retains the flat contact so that its proton pumping activity does not reduce much (Hackett et al., 1987; Mogi et al., 1987). 


\section{Ren: Isomerization sampling}

339

340

341

342

343

344

345

346

347

348

349

350

351

352

353

354

355

356

357

358

359

360

361

362

363

364

365

366

367

368

369

370

371

However, it is predictable that various single mutants at this position with smaller and smaller side chains would promote more and more isomerization byproducts and eventually shut down proton pumping.

Two massive side chains of Trp86 and 182 from the EC and CP sides respectively do not seem to play a significant role in suppressing byproduct formation as shown by the mutant W182F that retains the most of the wildtype activity (Hackett et al., 1987), since the motions involved in isomerization sampling are oriented more laterally. The transient expansion and contraction of the retinal binding pocket (Fig. $3 \mathrm{~d}$ ) indicate that the tight box surrounds the mid-segment of the retinal (Fig. 3e) is not completely rigid. Rather, its plasticity must carry sufficient strength to prevent isomerization byproducts. Presumably, this strength originates from the mechanical property of the helical bundle.

In summary, this work reveals the transient structural responses to many unsuccessful attempts of double bond isomerization and single bond rotation by a numerical resolution from the concurrent pathways, which are otherwise difficult to observe. These findings underscore an important implication, that is, a nonspecific Coulomb attraction provides the same driving force for the isomerization sampling with and without a protein matrix. A productive isomerization at a specific double bond is guided by the incorporation of the chromophore in a specific protein environment. The productive pathway is selected from numerous possibilities via stereochemical hinderance. Nevertheless, this nonspecific Coulomb attraction force may not be directly applicable to the photoisomerization of retinal from 11-cis to alltrans in the activation of visual rhodopsins. The key difference is $b R$ as an energy convertor versus a visual rhodopsin as a quantum detector (Lewis, 1978).

\section{Acknowledgements}

This work is supported in part by the grant R01EY024363 from National Institutes of Health. The following database and software are used in this work: CCP4 (ccp4.ac.uk), Coot (www2.mrc-lmb.cam.ac.uk/Personal/pemsley/coot), dynamiX ${ }^{\mathrm{TM}}$ (Renz Research, Inc.), gnuplot (gnuplot.info), PDB (rcsb.org), PHENIX (phenix-online.org), PyMOL (pymol.org), Python (python.org), and SciPy (scipy.org). 
Ren: Isomerization sampling

\section{Competing interests}

$\mathrm{ZR}$ is the founder of Renz Research, Inc. that currently holds the copyright of the

$$
\text { computer software dynami } X^{\mathrm{TM}} \text {. }
$$

\section{References}

Adams, P.D., Afonine, P.V., Bunkóczi, G., Chen, V.B., Davis, I.W., Echols, N., Headd, J.J., Hung, L.-W., Kapral, G.J., Grosse-Kunstleve, R.W., et al. (2010). PHENIX: a comprehensive Python-based system for macromolecular structure solution. Acta Crystallogr. D Biol. Crystallogr. D66, 213-221.

Altoè, P., Cembran, A., Olivucci, M., and Garavelli, M. (2010). Aborted double bicycle-pedal isomerization with hydrogen bond breaking is the primary event of bacteriorhodopsin proton pumping. Proc. Natl. Acad. Sci. 107, 20172-20177.

Ansari, A., Berendzen, J., Bowne, S.F., Frauenfelder, H., Iben, I.E., Sauke, T.B., Shyamsunder, E., and Young, R.D. (1985). Protein states and proteinquakes. Proc. Natl. Acad. Sci. 82, 5000-5004.

Brändén, G., and Neutze, R. (2021). Advances and challenges in time-resolved macromolecular crystallography. Science 373, eaba0954.

Chandonia, J.-M., and Brenner, S.E. (2006). The impact of structural genomics: expectations and outcomes. Science 311, 347-351.

Diller, R., Maiti, S., Walker, G.C., Cowen, B.R., Pippenger, R., Bogomolni, R.A., and Hochstrasser, R.M. (1995). Femtosecond time-resolved infrared laser study of the J-K transition of bacteriorhodopsin. Chem. Phys. Lett. 241, 109-115.

Ernst, O.P., Lodowski, D.T., Elstner, M., Hegemann, P., Brown, L.S., and Kandori, H. (2014). Microbial and animal rhodopsins: Structures, functions, and molecular mechanisms. Chem. Rev. 114, 126-163.

Freedman, K.A., and Becker, R.S. (1986). Comparative investigation of the photoisomerization of the protonated and unprotonated n-butylamine Schiff bases of 9-cis-, 11-cis-, 13-cis-, and all-trans-retinals. J. Am. Chem. Soc. 108, 1245-1251.

Glynn, C., and Rodriguez, J.A. (2019). Data-driven challenges and opportunities in crystallography. Emerg. Top. Life Sci. ETLS20180177.

Govindjee, R., Balashov, S.P., and Ebrey, T.G. (1990). Quantum efficiency of the photochemical cycle of bacteriorhodopsin. Biophys. J. 58, 597-608.

Hackett, N.R., Stern, L.J., Chao, B.H., Kronis, K.A., and Khorana, H.G. (1987). Structure-function studies on bacteriorhodopsin. V. Effects of amino acid substitutions in the putative helix F. J. Biol. Chem. 262, 9277-9284.

Henry, E.R., and Hofrichter, J. (1992). Singular value decomposition: Application to analysis of experimental data. In Numerical Computer Methods, (Academic Press), pp. 129-192.

Herbst, J. (2002). Femtosecond infrared spectroscopy of bacteriorhodopsin chromophore isomerization. Science 297, 822-825.

Joh, N.H., Min, A., Faham, S., Whitelegge, J.P., Yang, D., Woods, V.L., and Bowie, J.U. (2008). Modest stabilization by most hydrogen-bonded side-chain interactions in membrane proteins. Nature 453, 12661270. 


\section{Ren: Isomerization sampling}

411 Johnson, P.J.M., Halpin, A., Morizumi, T., S. Brown, L., I. Prokhorenko, V., P. Ernst, O., and Miller, R.J.D. 412 (2014). The photocycle and ultrafast vibrational dynamics of bacteriorhodopsin in lipid nanodiscs. Phys. 413 Chem. Chem. Phys. 16, 21310-21320.

414 Jung, Y.O., Lee, J.H., Kim, J., Schmidt, M., Moffat, K., Šrajer, V., and Ihee, H. (2013). Volume-conserving trans-cis isomerization pathways in photoactive yellow protein visualized by picosecond X-ray crystallography. Nat. Chem. 5, 212-220.

417 Kandori, H. (2015). Ion-pumping microbial rhodopsins. Front. Mol. Biosci. 2.

418 Kobayashi, T., Saito, T., and Ohtani, H. (2001). Real-time spectroscopy of transition states in

419 bacteriorhodopsin during retinal isomerization. Nature 414, 531-534.

420 Kovacs, G.N., Colletier, J.-P., Grünbein, M.L., Yang, Y., Stensitzki, T., Batyuk, A., Carbajo, S., Doak, R.B., 421 Ehrenberg, D., Foucar, L., et al. (2019). Three-dimensional view of ultrafast dynamics in photoexcited 422 bacteriorhodopsin. Nat. Commun. 10, 3177.

423 Koyama, Y., Kubo, K., Komori, M., Yasuda, H., and Mukai, Y. (1991). Effect of protonation on the 424 isomerization properties of n-butylamine Schiff base of isomeric retinal as revealed by direct HPLC 425 analyses: Selection of isomerization pathways by retinal proteins. Photochem. Photobiol. 54, 433-443.

Lanyi, J.K., and Schobert, B. (2007). Structural changes in the L photointermediate of bacteriorhodopsin. J.

Lewis, A. (1978). The molecular mechanism of excitation in visual transduction and bacteriorhodopsin. Proc. Natl. Acad. Sci. 75, 549-553. 238301.

433 Liebschner, D., Afonine, P.V., Baker, M.L., Bunkóczi, G., Chen, V.B., Croll, T.I., Hintze, B., Hung, L.-W., 434 Jain, S., McCoy, A.J., et al. (2019). Macromolecular structure determination using X-rays, neutrons and 435 electrons: recent developments in Phenix. Acta Crystallogr. Sect. Struct. Biol. 75, 861-877. translocation. J. Biol. Chem. 266, 6919-6927.

Mathies, R., and Stryer, L. (1976). Retinal has a highly dipolar vertically excited singlet state: implications for vision. Proc. Natl. Acad. Sci. 73, 2169-2173.

441 Mathies, R., Brito Cruz, C., Pollard, W., and Shank, C. (1988). Direct observation of the femtosecond 442 excited-state cis-trans isomerization in bacteriorhodopsin. Science 240, 777-779.

Mogi, T., Stern, L.J., Hackett, N.R., and Khorana, H.G. (1987). Bacteriorhodopsin mutants containing single tyrosine to phenylalanine substitutions are all active in proton translocation. Proc. Natl. Acad. Sci. $84,5595-5599$.

449 Perálvarez, A., Barnadas, R., Sabés, M., Querol, E., and Padrós, E. (2001). Thr90 is a key residue of the 450 bacteriorhodopsin proton pumping mechanism. FEBS Lett. 508, 399-402.

451 Ren, Z. (2013a). Reaction trajectory revealed by a joint analysis of Protein Data Bank. PLoS ONE 8, e77141. 


\section{Ren: Isomerization sampling}

452 Ren, Z. (2013b). Reverse engineering the cooperative machinery of human hemoglobin. PLoS ONE 8, 453 e77363.

454 Ren, Z. (2016). Molecular events during translocation and proofreading extracted from 200 static structures of DNA polymerase. Nucleic Acids Res. 6, 1-13.

456 Ren, Z. (2019). Ultrafast structural changes decomposed from serial crystallographic data. J. Phys. Chem. 457 Lett. 10, 7148-7163.

Ren, Z., Perman, B., Srajer, V., Teng, T.-Y., Pradervand, C., Bourgeois, D., Schotte, F., Ursby, T., Kort, R., Wulff, M., et al. (2001). A molecular movie at $1.8 \AA$ Å resolution displays the photocycle of photoactive yellow protein, a eubacterial blue-light receptor, from nanoseconds to seconds. Biochemistry 40, 1378813801.

Ren, Z., Chan, P.W.Y., Moffat, K., Pai, E.F., Royer, W.E., Šrajer, V., and Yang, X. (2013). Resolution of structural heterogeneity in dynamic crystallography. Acta Cryst D69, 946-959.

464 Schaffer, J.E., Kukshal, V., Miller, J.J., Kitainda, V., and Jez, J.M. (2021). Beyond X-rays: an overview of 465 emerging structural biology methods. Emerg. Top. Life Sci. ETLS20200272. charge translocation of photoexcited retinal in bacteriorhodopsin. Science 309, 917-920.

468 Schmidt, M., Rajagopal, S., Ren, Z., and Moffat, K. (2003). Application of singular value decomposition to the analysis of time-resolved macromolecular X-ray data. Biophys. J. 84, 2112-2129.

470 Schmidt, M., Graber, T., Henning, R., and Srajer, V. (2010). Five-dimensional crystallography. Acta

471 Crystallogr. A 66, 198-206.

472 Šrajer, V., Ren, Z., Teng, T.-Y., Schmidt, M., Ursby, T., Bourgeois, D., Pradervand, C., Schildkamp, W., Wulff, M., and Moffat, K. (2001). Protein conformational relaxation and ligand migration in myoglobin: A nanosecond to millisecond molecular movie from time-resolved Laue X-ray diffraction. Biochemistry 40, 13802-13815.

Tahara, S., Kuramochi, H., Takeuchi, S., and Tahara, T. (2019). Protein dynamics preceding photoisomerization of the retinal chromophore in bacteriorhodopsin revealed by deep-UV femtosecond stimulated Raman spectroscopy. J. Phys. Chem. Lett. 10, 5422-5427.

479 Ursby, T., and Bourgeois, D. (1997). Improved estimation of structure-factor difference amplitudes from 480 poorly accurate data. Acta Crystallogr. A 53, 564-575.

481 Wang, Q., Schoenlein, R.W., Peteanu, L.A., Mathies, R.A., and Shank, C.V. (1994). Vibrationally coherent 482 photochemistry in the femtosecond primary event of vision. Science 266, 422-424.

483 Yang, X., Ren, Z., Kuk, J., and Moffat, K. (2011). Temperature-scan cryocrystallography reveals reaction 484 intermediates in bacteriophytochrome. Nature 479, 428-432.

485 Zhong, Q., Ruhman, S., Ottolenghi, M., Sheves, M., Friedman, N., Atkinson, G.H., and Delaney, J.K. locked chromophore. J Am Chem Soc 118, 12828-12829. 


\section{Ren: Isomerization sampling}

\section{$492 \quad$ Figures and Legends}

493
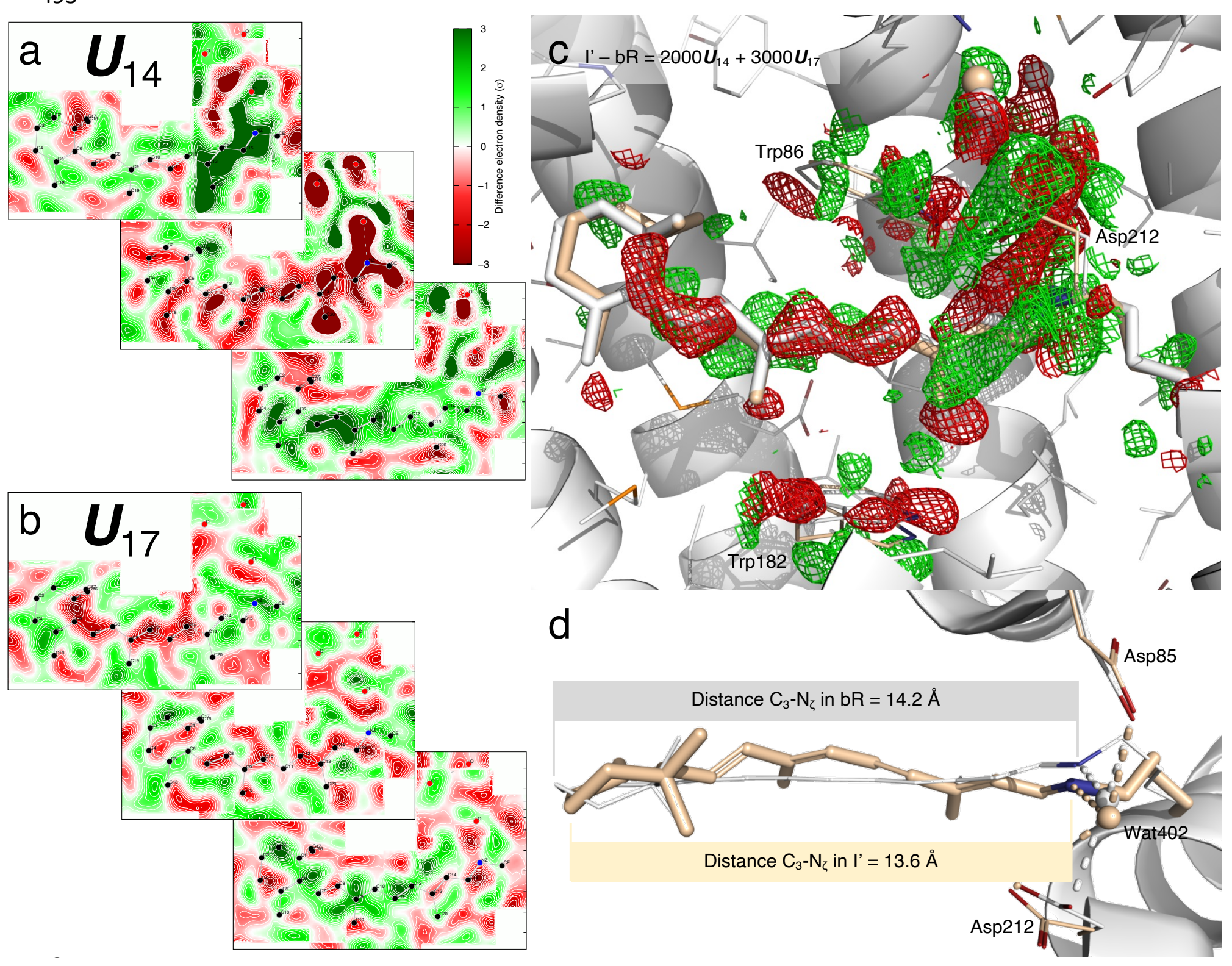

495

496 Figure 1. Shortened retinal in S-shape since earliest intermediate I'. (a) Cross sections

497 of component map $\boldsymbol{U}_{14}$. The middle cross section is an integration $\pm 0.2 \AA$ around the

498 surface through the retinal. The top cross section is an integration 1.2-1.8 $\AA$ outboard

499 from the retinal surface and the bottom one is an integration 0.8-1.2 A inboard. See Fig.

500 S1 for definitions of inboard, outboard, and other orientations in bR molecule. Green

501 and red indicate electron density gain and loss, respectively. Nearly the entire retinal is

502 in negative densities. The proximal segment and three waters are in intense negative 


\section{Ren: Isomerization sampling}

503 densities. On the other hand, strong positive densities flank the proximal and distal 504 segments from the outboard and inboard, respectively. Such signal distribution results 505 in the S-shaped retinal by the refinement shown in (d). (b) Cross sections of component 506 map $\boldsymbol{U}_{17}$. The middle cross section is an integration $\pm 0.2 \AA$ around the surface through 507 the retinal. The top panel is an integration 0.5-0.9 $\AA$ outboard and the bottom is an 508 integration 0.8-1.2 $\AA$ inboard. Negative and positive densities flank the retinal from the 509 outboard and inboard, respectively. (c) Difference map of $I^{\prime}-b R$ reconstituted from $\boldsymbol{U}_{14}$ 510 and $\boldsymbol{U}_{17}$ (a and b). The map is contoured at $\pm 3 \sigma$ in green and red mesh, respectively.

511 The opposite displacements of the distal and proximal segments of the retinal are

512 obvious. Extensive signals indicate changes in the water network and Asp85 and 212.

513 (d) Refined retinal conformation in beige overlaid on the resting state in white. This

514 view is orthographical to (c). The marked distances from $\mathrm{C}_{3}$ to $\mathrm{N}_{\zeta}$ show a shortened 515 retinal creased into an S-shape. $\mathrm{C}_{20}$ methyl group is tilted $33^{\circ}$ toward outboard from its 516 resting state bR. Wat402 remains in H-bonds with both Asp85 and 212. 
bioRxiv preprint doi: https://doi.org/10.1101/2021.09.16.460656; this version posted September 16, 2021. The copyright holder for this preprint (which was not certified by peer review) is the author/funder. All rights reserved. No reuse allowed without permission.

Ren: Isomerization sampling
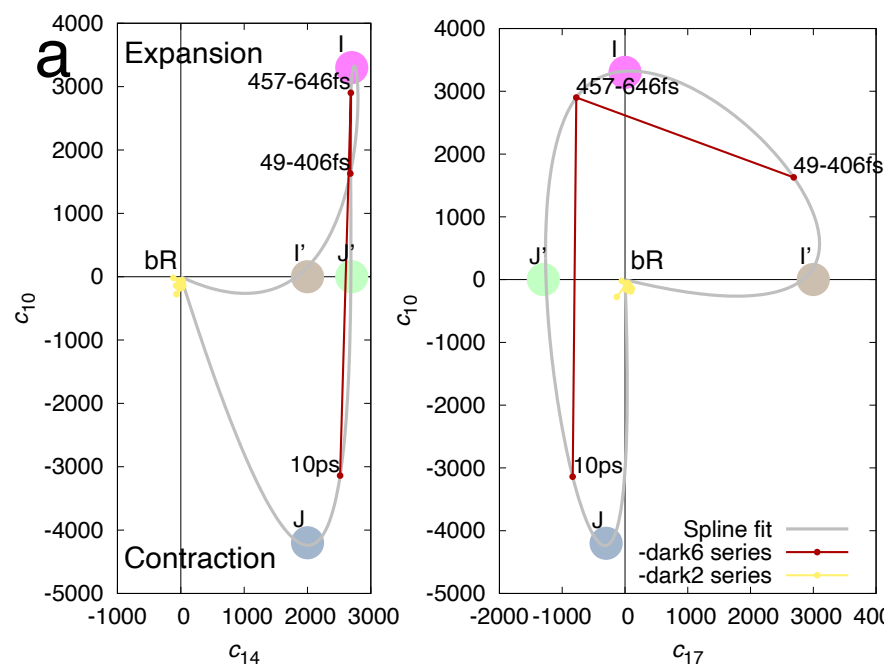

C
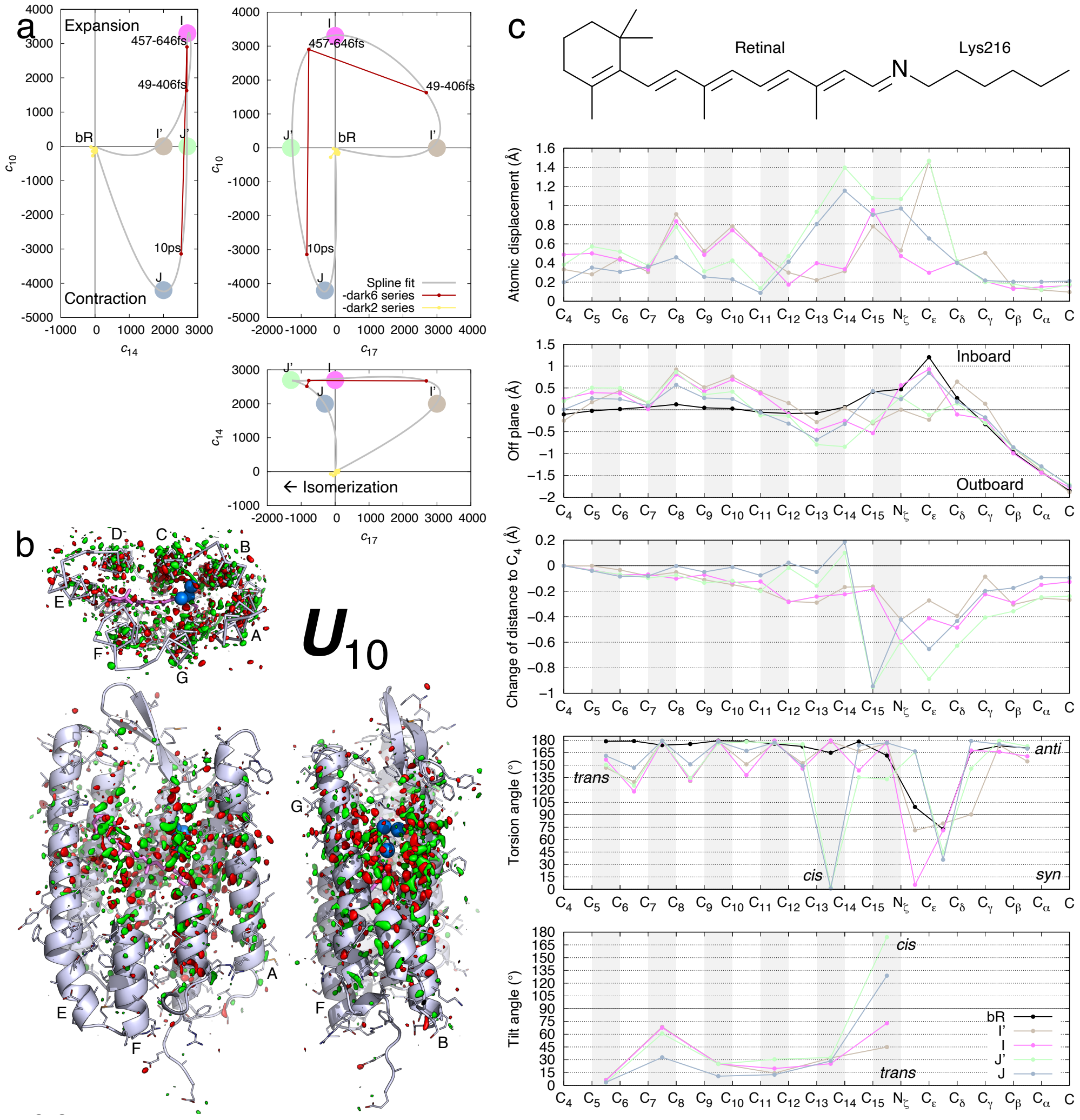

519 


\section{Ren: Isomerization sampling}

520 Figure 2. Early intermediates identified in SVD space. (a) Multi-dimensional spaces of 521 SVD. The SVD analysis of difference Fourier maps at short delays $\leq 10$ ps results in 522 time-dependent coefficients $c_{k}(t)$, where $k=1,2, \ldots$, each corresponding to a time-

523 independent components $\boldsymbol{U}_{k}$. Each raw difference map at a time delay $t$ can be closely 524 represented by a linear combination of these components, $c_{1}(t) \boldsymbol{U}_{1}+c_{2}(t) \boldsymbol{U}_{2}+\ldots$, that is

525 called a reconstituted difference map. Each of these components $\boldsymbol{U}_{k}$ and the

526 reconstituted difference maps can be rendered in the same way as an observed 527 difference map. The coefficient set $c_{k}(t)$ is therefore a trace of the photocycle trajectory, 528 when these time-dependent functions are plotted in a multi-dimensional space or 529 plotted together against the common variable $t$. Coefficients corresponding to 530 components $\boldsymbol{U}_{10}, \boldsymbol{U}_{14}$, and $\boldsymbol{U}_{17}$ are plotted in three orthographical views. Three time 531 points from Nogly et al. in red contain $\boldsymbol{U}_{14}$ equally. These time points vary in $\boldsymbol{U}_{10}$ and $532 U_{17}$. Datasets from Kovacs et al. in yellow do not carry any of these signals, therefore 533 cluster near the origin. The component map of $\boldsymbol{U}_{10}$ is displayed in (b) and $\boldsymbol{U}_{14}$ is 534 displayed in Figs. 1a and S5. $U_{17}$ is displayed in Fig. 1b. Several apices of the spline 535 fitting are chosen as the potential pure states of $\mathrm{I}^{\prime}, \mathrm{I}^{\prime} \mathrm{J}^{\prime}$, and J marked by large dots. This 536 choice is only an approximate due to the insufficient number of time points observed.

537 (b) Component map $\boldsymbol{U}_{10}$. The main chain and side chains of the protein are rendered 538 with ribbons and sticks, respectively. The retinal and Lys216 are in purple sticks.

539 Several key waters are in blue spheres. Parts of the structure are omitted to reveal more 540 of the interior. The map is contoured at $\pm 3 \sigma$ in green and red, respectively. Three 541 orthographical views of $\boldsymbol{U}_{10}$ clearly show that the signals are distributed around the 542 middle segment of the molecule and taper off to both CP and EC surfaces. The signals 543 also concentrate at all seven helices. (c) Conformational parameters calculated from the 544 refined chromophore. The chemical structure of the chromophore on top is aligned to 545 the horizontal axis. Double bonds are shaded in gray. Atomic displacements of each 546 intermediate from the resting state show greater changes in the proximal segment (top 547 panel). A plane is least-squares fitted to $\mathrm{C}_{4}$ through $\mathrm{C}_{14}$ of the resting state. The 548 distances of all atoms to this plane in the inboard and outboard directions show the 549 curvature of the chromophore. The creased retinal in early intermediates and the 550 inboard protruding corner at $C_{\varepsilon}$ in the resting state are clearly shown ( $2^{\text {nd }}$ panel). 551 Distances to atom $\mathrm{C}_{4}$ are calculated for all refined chromophores. Changes in these 552 distances with respect to the resting state show the shortened chromophore in I' and I. 553 Once isomerization to 13-cis occurs, the segment from $\mathrm{C}_{15}$ to $\mathrm{C}_{\delta}$ around the SB becomes 


\section{Ren: Isomerization sampling}

554 significantly closer to the $\beta$-ionone ring due to the Coulomb attraction force, while the 555 distal segment of the retinal from $\mathrm{C}_{14}$ and beyond stretches ( $3^{\text {rd }}$ panel). The torsion

556 angles of single and double bonds quantify anti/syn or trans/cis for the ground state and 557 all intermediates ( $4^{\text {th }}$ panel). Only a single bond can be twisted with its torsion angle 558 near $90^{\circ}$. A twisted double bond would be energetically costly. Each double bond is 559 least-squares fitted with a plane. The interplanar angle between a double bond and the 560 corresponding one in the ground state measures the local tilting of the retinal (bottom 561 panel).

562

563 


\section{Ren: Isomerization sampling}

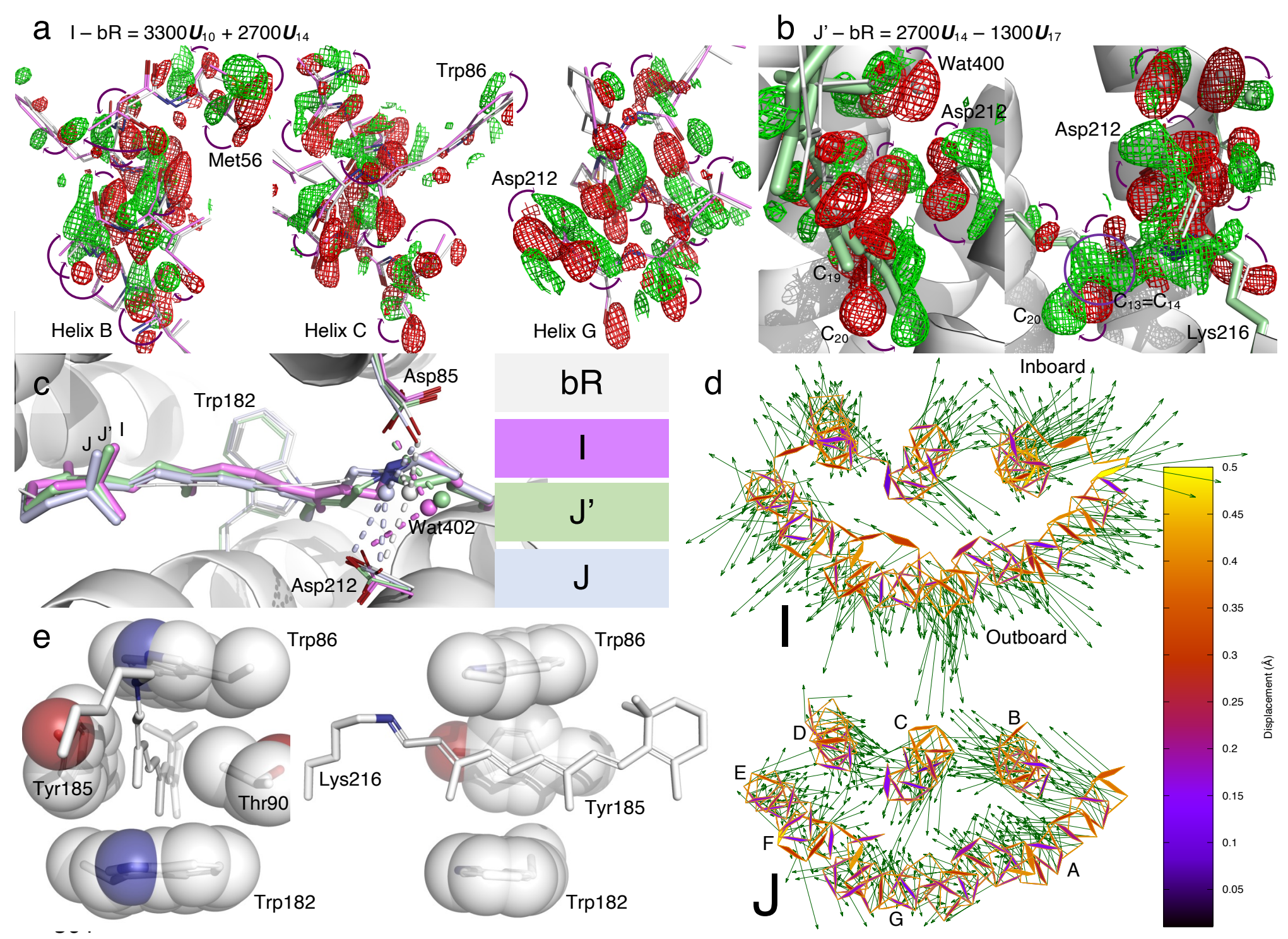

565

566 Figure 3. Intermediates I, J', J, and retinal binding pocket expansion and contraction.

567 (a) Reconstituted difference map I - bR from $\boldsymbol{U}_{10}$ and $\boldsymbol{U}_{14}$ (Figs. 1a, 2ab, and S5). The

568 map is contoured at $\pm 2.5 \sigma$ in green and red mesh, respectively. The difference map at

569 three middle segments of helices B, C, and G show main chain displacements toward

570 inboard or outboard as indicated by the arrows marking the negative and positive pairs

571 of densities. These difference densities are the direct evidence of the expansion of the

572 retinal binding pocket. The refined structure of $\mathrm{I}$ is in purple and the resting state is in

573 white. (b) Reconstituted difference map J' - bR from $\boldsymbol{U}_{14}$ and $\boldsymbol{U}_{17}$ (Figs. 1ab, 2a, and S5).

574 The map is contoured at $\pm 3.5 \sigma$ in green and red mesh, respectively. These difference

575 densities are the direct evidence of isomerization at hundreds of $\mathrm{fs}$. The refined

576 structure of J' in 13-cis is in green. (c) The refined 13-cis retinal conformation compared 


\section{Ren: Isomerization sampling}

577 with the resting state in white. I, J', and J are in purple, green, and bluish gray,

578 respectively. The creased S-shape is easing gradually (Fig. 2c $2^{\text {nd }}$ panel). (d) The refined

579 structures of I and J compared with the resting state viewed along the trimer three-fold

580 axis from the EC side. Atomic displacements in the main chain from $\mathrm{bR}$ to I and J are

581 color coded and marked by arrows with lengths $20 \times$ of the actual displacements. All

582 seven helices in I move away from the center except a small segment in helix C showing

583 an expansion of the retinal binding pocket (top panel). However, all seven helices in J

584 move closer to one another showing a contraction with respect to the resting state $\mathrm{bR}$

585 (bottom panel). This contraction is much more significant if compared directly with the

586 expanded I state. (e) Two orthographical views of the retinal tightly boxed at its middle

587 segment. The closest contact is Thr90 and Tyr185 on the inboard and outboard sides of

588 the retinal plane, respectively. The minimum distance between them is $7.0 \AA=4 r_{C}+0.2$

$589 \AA$, where $r_{C}=1.7 \AA$ is the van der Waals radius of C. See also (Kandori, 2015).

590

591 


\section{Photoinduced Isomerization Sampling of Retinal in}

\section{Bacteriorhodopsin}

595 Zhong Ren

Department of Chemistry, University of Illinois at Chicago, Chicago, IL 60607, USA

zren@uic.edu

\section{Methods}

604 From the outset, the key presumption is that every crystallographic dataset, at a given

605 temperature and a given time delay after the triggering of a photochemical reaction,

606 captures a mixture of unknown number of intermediate species at unknown fractions.

607 Needless to say, all structures of the intermediates are also unknown except the

608 structure at the ground state that has been determined and well refined by static

609 crystallography. A simultaneous solution of all these unknowns requires multiple

610 datasets that are collected at various temperatures or time delays so that a common set

611 of intermediate structures are present in these datasets with variable ratios. If the

612 number of available datasets is far greater than the number of unknowns, a linear

613 system can be established to overdetermine the unknowns with the necessary

614 stereochemical restraints (Ren et al., 2013). The analytical methods used in this work to

615 achieve such overdetermination have been incrementally developed in the past years

616 and recently applied to another joint analysis of the datasets of carbonmonoxy

617 myoglobin (Ren, 2019). Time-resolved datasets collected with ultrashort pulses from an

618 X-ray free electron laser were successfully analyzed by these methods to visualize

619 electron density components that reveal transient heating, $3 d$ electrons of the heme iron, 620 and global vibrational motions. This analytical strategy is recapped below.

622 The methodological advance in this work is the refinement of each pure

623 intermediate structure that has been deconvoluted from multiple mixtures. Structure

624 factor amplitudes of a single conformation free of heterogeneity are overdetermined. 


\section{Ren: Isomerization sampling}

625 Given the deconvoluted structure factor amplitude set of a pure state, the standard 626 structural refinement software with the built-in stereochemical constraints is taken full 627 advantage of, e.g. PHENIX (Adams et al., 2010; Liebschner et al., 2019). In case that the 628 computed deconvolution has not achieved a single pure structural species, the 629 structural refinement is expected to make such indication.

\section{Difference Fourier maps}

632 A difference Fourier map is synthesized from a Fourier coefficient set of $F_{\text {light }}-F_{\text {reference }}$ 633 with the best available phase set, often from the ground state structure. Before Fourier 634 synthesis, $F_{\text {light }}$ and $F_{\text {reference }}$ must be properly scaled to the same level so that the 635 distribution of difference values is centered at zero and not skewed either way. A 636 weighting scheme proven effective assumes that a greater amplitude of a difference 637 Fourier coefficient $F_{\text {light }}-F_{\text {reference }}$ is more likely caused by noise than by signal (Ren et al., 638 2001, 2013; Šrajer et al., 2001; Ursby and Bourgeois, 1997). Both the dark and light 639 datasets can serve as a reference in difference maps. If a light dataset at a certain delay 640 is chosen as a reference, the difference map shows the changes since that delay time but 641 not the changes prior to that delay. However, both the dark and light datasets must be

642 collected in the same experiment. A cross reference from a different experimental

643 setting usually causes large systematic errors in the difference map that would swamp 644 the desired signals. Each difference map is masked $3.5 \AA$ around the entire molecule of 645 bacteriorhodopsin (bR). No lipid density is analyzed.

Meta-analysis of protein structures

648 Structural meta-analysis based on singular value decomposition (SVD) has been 649 conducted in two forms. In one of them, an interatomic distance matrix is calculated 650 from each protein structure in a related collection. SVD of a data matrix consists of 651 these distance matrices enables a large-scale joint structural comparison but requires no 652 structural alignment (Ren, 2013a, 2013b, 2016). In the second form, SVD is performed 653 on a data matrix of electron densities of related protein structures (Ren, 2019; Ren et al., 654 2013; Schmidt et al., 2003, 2010). Both difference electron density maps that require a 655 reference dataset from an isomorphous crystal form and simulated annealing omit 656 maps that do not require the same unit cell and space group of the crystals are possible 657 choices in a structural meta-analysis (Ren, 2019; Ren et al., 2013). The interatomic 658 distances or the electron densities that SVD is performed on are called core data. Each 


\section{Ren: Isomerization sampling}

659 distance matrix or electron density map is associated with some metadata that describe

660 the experimental conditions under which the core data are obtained, such as

661 temperature, $\mathrm{pH}$, light illumination, time delay, mutation, etc. These metadata do not

662 enter the SVD procedure. However, they play important role in the subsequent

663 interpretation of the SVD result. This computational method of structural analysis takes

664 advantage of a mathematical, yet practical, definition of conformational space with

665 limited dimensionality (Ren, 2013a). Each experimentally determined structure is a

666 snapshot of the protein structure. A large number of such snapshots taken under a

667 variety of experimental conditions, the metadata, would collectively provide a survey of

668 the accessible conformational space of the protein structure and reveal its rection

669 trajectory. Such joint analytical strategy would not be effective in early years when far

670 fewer protein structures were determined to atomic resolution. Recent rapid growth in

671 protein crystallography, such as in structural genomics (Chandonia and Brenner, 2006,

672 2012) and in serial crystallography (Glynn and Rodriguez, 2019; Schaffer et al., 2021),

673 has supplied the necessarily wide sampling of protein structures for a joint analytical

674 strategy to come of age. The vacancies or gaps in a conformational space between well-

675 populated conformational clusters often correspond to less stable transient states whose

676 conformations are difficult to capture, if not impossible. These conformations are often

677 key to mechanistic understanding and could be explored by a back calculation based on

678 molecular distance geometry (Ren, 2013a, 2016), the chief computational algorithm in

679 nucleic magnetic resonance spectroscopy (NMR), and by a structure refinement based

680 on reconstituted dataset, a major methodological advance in this work (see below).

681 These structures refined to atomic resolution against reconstituted datasets may reveal

682 short-lived intermediate conformation hard to be captured experimentally.

683 Unfortunately, a protein structure refined against a reconstituted dataset currently

684 cannot be recognized by the Protein Data Bank (PDB). Because crystallographic

685 refinement of a macromolecular structure is narrowly defined as a correspondence from

686 one dataset to one structure. A never-observed dataset reconstituted from a collection

687 of experimental datasets does not match the well-established crystallographic template

688 of PDB; let alone a refinement of crystal structure with the NMR algorithm.

689

690

A distance matrix contains $M$ pairwise interatomic distances of a structure in the

691 form of Cartesian coordinates of all observed atoms. An everyday example of distance

692 matrix is an intercity mileage chart appended to the road atlas. Differences in the 


\section{Ren: Isomerization sampling}

693 molecular orientation, choice of origin, and crystal lattice among all experimentally

694 determined structures have no contribution to the distance matrices. Due to its

695 symmetry, only the lower triangle is necessary. A far more intimate examination of

696 protein structures in PDB is a direct analysis of their electron density maps instead of

697 the atomic coordinates. $M$ such (difference) electron densities, often called voxels in

698 computer graphics, are selected by a mask of interest. In the case of difference maps,

699 only the best refined protein structure in the entire collection supplies a phase set for

700 Fourier synthesis of electron density maps. This best structure is often the ground state

701 structure determined by static crystallography. Other refined atomic coordinates from

702 the PDB entries are not considered in the meta-analysis. That is to say, a meta-analysis

703 of difference electron density maps starts from the X-ray diffraction data archived in

704 PDB rather than the atomic coordinates interpreted from the diffraction data, which

705 removes any potential model bias.

706

707 Singular value decomposition of (difference) electron density maps

708 An electron density map, particularly a difference map as emphasized here, consists of

709 density values on an array of grid points within a mask of interest. All $M$ grid points in

710 a three-dimensional map can be serialized into a one-dimensional sequence of density

711 values according to a specific protocol. It is not important what the protocol is as long

712 as a consistent protocol is used to serialize all maps of the same grid setting and size,

713 and a reverse protocol is available to erect a three-dimensional map from a sequence of

$714 M$ densities. Therefore, a set of $N$ serialized maps, also known as vectors in linear

715 algebra, can fill the columns of a data matrix $\mathbf{A}$ with no specific order, so that the width

716 of $\mathbf{A}$ is $N$ columns, and the length is $M$ rows. Often, $M \gg N$, thus $\mathbf{A}$ is an elongated

717 matrix. If a consistent protocol of serialization is used, the corresponding voxel in all $N$

718 maps occupies a single row of matrix A. This strict correspondence in a row of matrix

719 A is important. Changes of the density values in a row from one structure to another

720 are due to either signals, systematic errors, or noises. Although the order of columns in

721 matrix $\mathbf{A}$ is unimportant, needless to say, the metadata associated with each column

722 must remain in good bookkeeping.

723

724 SVD of the data matrix $\mathbf{A}$ results in $\mathbf{A}=\mathbf{U W V}^{\mathrm{T}}$, also known as matrix factorization.

725 Matrix $\mathbf{U}$ has the same shape as $\mathbf{A}$, that is, $N$ columns and $M$ rows. The $N$ columns

726 contain decomposed basis components $\boldsymbol{U}_{k}$, known as left singular vectors of $M$ items, 


\section{Ren: Isomerization sampling}

727 where $k=1,2, \ldots, N$. Therefore, each component $\boldsymbol{U}_{k}$ can be erected using the reverse 728 protocol to form a three-dimensional map. This decomposed elemental map can be 729 presented in the same way as the original maps, for example, rendered in molecular 730 graphics software such as Coot and PyMol. It is worth noting that these decomposed 731 elemental maps or map components $\boldsymbol{U}_{k}$ are independent of any metadata. That is to 732 say, these components remain constant when the metadata vary. Since each left 733 singular vector $\boldsymbol{U}_{k}$ has a unit length due to the orthonormal property of SVD (see 734 below), that is, $\left|\boldsymbol{U}_{k}\right|=1$, the root mean squares (rms) of the items in a left singular 735 vector is $1 / \sqrt{ } M$ that measures the quadratic mean of the items.

The second matrix $\mathbf{W}$ is a square matrix that contains all zeros except for $N$ positive 738 values on its major diagonal, known as singular values $w_{k}$. The magnitude of $w_{k}$ is 739 considered as a weight or significance of its corresponding component $\boldsymbol{U}_{k}$. The third 740 matrix $\mathbf{V}$ is also a square matrix of $N \times N$. Each column of $\mathbf{V}$ or row of its transpose $\mathbf{V}^{\mathrm{T}}$, 741 known as a right singular vector $\boldsymbol{V}_{k}$, contains the relative compositions of $\boldsymbol{U}_{k}$ in each of 742 the $N$ original maps. Therefore, each right singular vector $V_{k}$ can be considered as a 743 function of the metadata. Right singular vectors also have the same unit length, that is, $744\left|V_{k}\right|=1$. Effectively, SVD separates the constant components independent of the 745 metadata from the compositions that depend on the metadata. A singular triplet denotes 1) a decomposed component $\boldsymbol{U}_{k,}$ 2) its singular value $w_{k}$, and 3 ) the composition function $\boldsymbol{V}_{k}$. Singular triplets are often sorted in a descending order of their singular values $w_{k}$. Only a small number of $n$ significant singular triplets identified by the greatest singular values $w_{1}$ through $w_{n}$ can be used in a linear combination to reconstitute a set of composite maps that closely resemble the original ones in matrix $\mathbf{A}$, where $n<N$. For example, the original map in the $i$ th column of matrix A under a certain experimental condition can be closely represented by the $i$ th composite map $w_{1} v_{1 i} \boldsymbol{U}_{1}+w_{2} v_{2 i} \boldsymbol{U}_{2}+\ldots+w_{n} v_{n i} \boldsymbol{U}_{n}$, where $\left(v_{1 i}, v_{2 i}, \ldots\right)$ is from the $i$ th row of matrix $\mathbf{V}$. The coefficient set for the linear combination is redefined here as $c_{k i}=$ $w_{k} v_{k i} / \sqrt{ } M$. The rms of the density values in a map component, or the average magnitude measured by the quadratic mean, acts as a constant scale factor that resets the modified coefficients $c_{k i}$ back to the original scale of the core data, such as $\AA$ for distance matrices 


\section{Ren: Isomerization sampling}

761 calibration, which makes this scale factor unnecessary. In the linear combination $c_{1 i} \boldsymbol{U}_{1}+$

$762 \mathcal{C}_{2 i} \boldsymbol{U}_{2}+\ldots+\boldsymbol{C}_{n i} \boldsymbol{U}_{n}$, each component $\boldsymbol{U}_{k}$ is independent of the metadata while how much of 763 each component is required for the approximation, that is, $c_{k i}$, depends on the metadata.

Excluding the components after $\boldsymbol{U}_{n}$ in this approximation is based on an assumption that the singular values after $w_{n}$ are very small relative to those from $w_{1}$ through $w_{n}$. As a result, the structural information evenly distributed in all $N$ original maps is effectively concentrated into a far fewer number of $n$ significant components, known as information concentration or dimension reduction. On the other hand, the trailing components in matrix $\mathbf{U}$ contain inconsistent fluctuations and random noises.

772 squares property of SVD guarantees that the rejected trailing components sums up to

773 the least squares of the discrepancies between the original core data and the

774 approximation using the accepted components.

However, no clear boundary is guaranteed between signals, systematic errors, and 778 excluding some components from 1 through $n$ is also possible. If systematic errors are 779 correctly identified, the reconstituted map without these significant components would no longer carry the systematic errors.

The orthonormal property of SVD

783 The solution set of SVD must guarantee that the columns in $\mathbf{U}$ and $\mathbf{V}$, the left and right 784 singular vectors $\boldsymbol{U}_{k}$ and $\boldsymbol{V}_{k}$, are orthonormal, that is, $\boldsymbol{U}_{h} \bullet \boldsymbol{U}_{k}=\boldsymbol{V}_{h} \bullet \boldsymbol{V}_{k}=0$ (ortho) and $\boldsymbol{U}_{k} \bullet \boldsymbol{U}_{k}$ $785=V_{k} \bullet V_{k}=1$ (normal), where $h \neq k$ but both are from 1 to $N$. The orthonormal property 786 also holds for the row vectors. As a result, each component $\boldsymbol{U}_{k}$ is independent of the 787 other components. In other words, a component cannot be represented by a linear 788 combination of any other components. However, two physical or chemical parameters 789 in the metadata, such as temperature and $\mathrm{pH}$, may cause different changes to a 790 structure. These changes are not necessarily orthogonal. They could exhibit some 791 correlation. Therefore, the decomposed components $\boldsymbol{U}_{k}$ not necessarily represent any 792 physically or chemically meaningful changes (see below). 


\section{Ren: Isomerization sampling}

Due to the orthonormal property of SVD, an N-dimensional Euclidean space is established, and the first $n$ dimensions define its most significant subspace. Each coefficient set $c_{i}=\left(c_{1 i}, c_{2} i, \ldots, c_{n i}\right)$ of the $i$ th composite map is located in this $n$-dimensional subspace. All coefficient sets for $i=1,2, \ldots, N$ in different linear combinations to approximate the $N$ original maps in a least-squares sense can be represented by $N$ points or vectors $c_{1}, c_{2}, \ldots, c_{N}$ in the Euclidean subspace. This $n$-dimensional subspace is essentially the conformational space as surveyed by the jointly analyzed core data. The conformational space is presented as scatter plots with each captured structure represented as a dot located at a position determined by the coefficient set $c_{i}$ of the $i$ th observed map. When the subspace has greater dimensionality than two, multiple twodimensional orthographical projections of the subspace are presented, such as Fig. 2a. These scatter plots are highly informative to reveal the relationship between the (difference) electron density maps and their metadata.

If two coefficient sets $\boldsymbol{c}_{i} \approx \boldsymbol{c}_{j}$, they are located close to each other in the conformational space. Therefore, these two structures $i$ and $j$ share two similar conformations. Two structures located far apart from each other in the conformational space are dissimilar in their conformations, and distinct in the compositions of the map components. A reaction trajectory emerges in this conformational space if the temporal order of the core data is experimentally determined (Fig. 2a). Otherwise, an order could be assigned to these structures based on an assumed smoothness of conformational changes along a reaction trajectory (Ren, 2013a, 2013b, 2016). Causation and consequence of structural motions could be revealed from the order of the structures in a series, which may further lead to structural mechanism. In addition, an off-trajectory location in the conformational space or a location between two clusters of observed structures represents a structure in a unique conformation that has never been experimentally captured. Such a hypothetical structure can be refined against a reconstituted distance matrix using molecular distance geometry (Ren, 2013a, 2013b, 2016) or a reconstituted electron density map with the method proposed below.

\section{Rotation in SVD space}

Dimension reduction is indeed effective in meta-analysis of protein structures when many datasets are evaluated at the same time. However, the default solution set of SVD carries complicated physical and chemical meanings that are not immediately obvious. 


\section{Ren: Isomerization sampling}

828 The interpretation of a basis component $\boldsymbol{U}_{k}$, that is, "what-does-it-mean", requires a 829 clear demonstration of the relationship between the core data and their metadata. The 830 outcome of SVD does not guarantee any physical meaning in a basis component.

831 Therefore, SVD alone provides no direct answer to "what-does-it-mean", thus its 832 usefulness is very limited to merely a mathematical construction. However, the

833 factorized set of matrices $\mathbf{U}, \mathbf{W}$, and $\mathbf{V}$ from SVD is not a unique solution. That is to say,

834 they are not the only solution to factorize matrix A. Therefore, it is very important to 835 find one or more alternative solution sets that are physically meaningful to elucidate a 836 structural interpretation. The concept of a rotation after SVD was introduced by Henry 837 \& Hofrichter (Henry and Hofrichter, 1992). But they suggested a protocol that fails to 838 preserve the orthonormal and least-squares properties of SVD. The rotation protocol 839 suggested by Ren incorporates the metadata into the analysis and combines with SVD 840 of the core data. This rotation achieves a numerical deconvolution of multiple physical 841 and chemical factors after a pure mathematical decomposition, and therefore, provides 842 a route to answer the question of "what-does-it-mean" (Ren, 2019). This rotation shall

843 not be confused with a rotation in the three-dimensional real space, in which a

844 molecular structure resides.

A rotation in the $n$-dimensional Euclidean subspace is necessary to change the perspective before a clear relationship emerges to elucidate scientific findings. It is shown below that two linear combinations are identical before and after a rotation applied to both the basis components and their coefficients in a two-dimensional subspace of $h$ and $k$. That is,

853 where $c_{h}$ and $c_{k}$ are the coefficients of the basis components $\boldsymbol{U}_{h}$ and $\boldsymbol{U}_{k}$ before the 854 rotation; and $f_{h}$ and $f_{k}$ are the coefficients of the rotated basis components $\boldsymbol{R}_{h}$ and $\boldsymbol{R}_{k}$, 855 respectively. The same Givens rotation of an angle $\theta$ is applied to both the components 856 and their coefficients:

857

$$
\left\{\begin{array}{l}
\boldsymbol{R}_{h}=\boldsymbol{U}_{h} \cos \theta-\boldsymbol{U}_{k} \sin \theta \\
\boldsymbol{R}_{k}=\boldsymbol{U}_{h} \sin \theta+\boldsymbol{U}_{k} \cos \theta
\end{array}\right.
$$




\section{Ren: Isomerization sampling}

859 Obviously, the rotated components $\boldsymbol{R}_{h}$ and $\boldsymbol{R}_{k}$ remain mutually orthonormal and orthonormal to other components. And

861

862

$$
\left\{\begin{array}{l}
f_{h}=s_{h} t_{h}=c_{h} \cos \theta-c_{k} \sin \theta \\
f_{k}=s_{k} t_{k}=c_{h} \sin \theta+c_{k} \cos \theta
\end{array}\right.
$$

863 Here $s_{h \mid k}=\sqrt{\sum f_{h \mid k}^{2}}$ are the singular values that replace $w_{h}$ and $w_{k}$, respectively, after the 864 rotation. They may increase or decrease compared to the original singular values so 865 that the descending order of the singular values no longer holds. $T_{h \mid k}=\left(t_{h \mid k 1}, t_{h \mid k 2, \ldots}\right.$, $\left.866 t_{h \mid k N}\right)=\left(f_{h \mid k 1}, f_{h \mid k 2}, \ldots, f_{h \mid k N}\right) / s_{h \mid k}$ are the right singular vectors that replace $\boldsymbol{V}_{h}$ and $\boldsymbol{V}_{k}$,

867 respectively. $\boldsymbol{T}_{h}$ and $\boldsymbol{T}_{k}$ remain mutually orthonormal after the rotation and 868 orthonormal to other right singular vectors that are not involved in the rotation.

To prove Eq. 1, Eqs. 2 and 3 are combined and expanded. All cross terms of sine and cosine are self-canceled:

872

$$
f_{h} \boldsymbol{R}_{h}+f_{k} \boldsymbol{R}_{k}=\left(c_{h} \cos \theta-c_{k} \sin \theta\right)\left(\boldsymbol{U}_{h} \cos \theta-\boldsymbol{U}_{k} \sin \theta\right)+\left(c_{h} \sin \theta+c_{k} \cos \theta\right)\left(\boldsymbol{U}_{h} \sin \theta+\boldsymbol{U}_{k} \cos \theta\right)
$$

$$
\begin{aligned}
& =c_{h} \boldsymbol{U}_{h} \cos ^{2} \theta+c_{k} \boldsymbol{U}_{k} \sin ^{2} \theta+c_{h} \boldsymbol{U}_{h} \sin ^{2} \theta+c_{k} \boldsymbol{U}_{k} \cos ^{2} \theta \pm c_{h} \boldsymbol{U}_{k} \sin \theta \cos \theta \pm c_{k} \boldsymbol{U}_{h} \sin \theta \cos \theta \\
& =c_{h} \boldsymbol{U}_{h}\left(\cos ^{2} \theta+\sin ^{2} \theta\right)+c_{k} \boldsymbol{U}_{\mathrm{k}}\left(\sin ^{2} \theta+\cos ^{2} \theta\right)
\end{aligned}
$$

$$
=c_{h} \boldsymbol{U}_{h}+c_{k} \boldsymbol{U}_{k}
$$

A rotation in two-dimensional subspace of $h$ and $k$ has no effect in other dimensions, as the orthonormal property of SVD guarantees. Multiple steps of rotations can be carried out in many two-dimensional subspaces consecutively to achieve a multidimensional rotation. A new solution set derived from a rotation retains the orthonormal property of SVD. The rotation in the Euclidean subspace established by SVD does not change the comparison among the core data of protein structures. Rather it converts one solution set $\mathbf{A}=\mathbf{U W V}^{\mathrm{T}}$ to other alternative solutions $\mathbf{A}=\mathbf{R S T}^{\mathrm{T}}$ so that an appropriate perspective can be found to elucidate the relationship between the core data and metadata clearly and concisely.

For example, if one physical parameter could be reoriented along a single dimension 890 $k$ but not involving other dimensions by a rotation, it can be convincingly shown that the left singular vector $\boldsymbol{U}_{k}$ of this dimension illustrates the structural impact by this 


\section{Ren: Isomerization sampling}

891 physical parameter. Before this rotation, the same physical parameter may appear to

892

893

894

895

896

897

898

899

900

901

902

903

904

905

906

907

908

909

910

911

912

913

914

915

916

917

918

919

920

921

922

923

924

cause structural variations along several dimensions, which leads to a difficult interpretation. Would a proper rotation establish a one-on-one correspondence from all physical or chemical parameters to all the dimensions? It depends on whether each parameter induces an orthogonal structural change, that is, whether structural responses to different parameters are independent or correlated among one another. If structural changes are indeed orthogonal, it should be possible to find a proper rotation to cleanly separate them in different dimensions. Otherwise, two different rotations are necessary to isolate two correlated responses, but one at a time.

For another example, if the observed core datasets form two clusters in the conformational space, a rotation would be desirable to separate these clusters along a single dimension $k$ but to align these clusters along other dimensions. Therefore, the component $\boldsymbol{U}_{k}$ is clearly due to the structural transition from one cluster to the other. Without a proper rotation, the difference between these clusters could be complicated with multiple dimensions involved. A deterministic solution depends on whether a clear correlation exists between the core data and metadata. A proper rotation may require a user decision. A wrong choice of rotation may select a viewpoint that hinders a concise conclusion. However, it would not alter the shape of the reaction trajectory, nor create or eliminate an intrinsic structural feature. A wrong choice of rotation cannot eliminate the fact that a large gap exists between two clusters of observed core datasets except that these clusters are not obvious from that viewpoint. A different rotation may reorient the perspective along another direction. But the structural conclusion would be equivalent. See example of before and after a rotation in (Ren, 2016).

This rotation procedure finally connects the core crystallographic datasets to the metadata of experimental conditions and accomplishes the deconvolution of physical or chemical factors that are not always orthogonal to one another after a mathematical decomposition. SVD analysis presented in this paper employs rotations extensively except that no distinction is made in the symbols of components and coefficients before and after a rotation except in this section. This method is widely applicable in largescale structural comparisons. Furthermore, Ren rotation after SVD is not limited to crystallography and may impact other fields wherever SVD is used. For example, SVD is frequently applied to spectroscopic data, images, and genetic sequence data. 


\section{Ren: Isomerization sampling}

925

926

927

928

929

930

931

932

933

934

935

936

937

938

939

940

941

942

943

944

945

946

947

948

949

950

951

952

953

954

955

956

957

958

\section{Structural refinement against reconstituted dataset}

The linear combination $\Delta \rho(t)=f_{1}(t) \boldsymbol{R}_{1}+f_{2}(t) \boldsymbol{R}_{2}+\ldots+f_{n}(t) \boldsymbol{R}_{n}$ after a rotation reconstitutes one of the observed difference maps at a specific time point $t$. This time-dependent difference map depicts an ever-evolving mixture of many excited species. A reconstituted difference map $\Delta \rho(\mathrm{E})$ for a time-independent, pure, excited species $\mathrm{E}=$ intermediate I', I, J', and J deconvoluted from many mixtures would take the same form except that only one or very few coefficients remain nonzero if a proper rotation has been found (Table S2). In order to take advantage of the mature refinement software for macromolecular structures with extensive stereochemical restraints, a set of structure factor amplitudes is needed. Therefore, it is necessary to reconstitute a set of structure factor amplitudes that would produce the target difference map $\Delta \rho(\mathrm{E})$ based on a known structure at the ground state. First, an electron density map of the structure at the ground state is calculated. This calculated map is used as a base map. Second, this base map of the ground state is combined with the positive and negative densities in the target difference map $\Delta \rho(\mathrm{E})$ so that the electron densities at the ground state are skewed toward the intermediate state. Third, structure factors are calculated from the combined map. Finally, the phase set of the calculated structure factors is discarded, and the amplitudes are used to refine a single conformation of the intermediate species E that $\Delta \rho(\mathrm{E})$ represents.

This protocol following the SVD and Ren rotation of components achieves a refinement of a pure structural species without the need of alternative conformations. Several points are noteworthy. First, the minimization protocol in this refinement is performed against a numerically reconstituted amplitude set that has never been directly measured from a crystal. This reconstituted dataset could be considered as an extrapolated dataset "on steroids" if compared to the traditional extrapolation of small differences, such as, the Fourier coefficient set to calculate a 3Fo-2Fc map, a technique often used to overcome a partial occupancy of an intermediate structure. An extrapolation of small differences is not directly observed either but computed by an exaggeration of the observed difference based on an assumption that the intermediate state is partially occupied, such as the doubling of the observed difference in $3 \mathrm{Fo}-2 \mathrm{Fc}=$ $\mathrm{Fo}+2(\mathrm{Fo}-\mathrm{Fc})$. In contrast to the conventional technique of extrapolation, the deconvolution method applied here is an interpolation among many experimental 
Ren: Isomerization sampling

959 datasets rather than an extrapolation. Secondly, the deconvolution is a simultaneous

960 solution of multiple intermediate states mixed together instead of solving a single

961 excited state.

962

Second, a map calculated from the ground state structure is chosen as the base map instead of an experimental map such as Fo or 2Fo-Fc map. If the second step of the protocol is skipped, that is, no difference map is combined with the ground state map, the refinement would result in an $R$ factor of nearly zero, since the refinement is essentially against the calculated structure factors (bR in Table S2). This is to say, the residuals of the refinement are solely due to the difference component instead of the base map. This is desirable since errors in the static structure of the ground state are gauged during its own refinement. On the other hand, if an experimental map is chosen as a base map, the refinement $R$ factors would reflect errors in both the base map

973 refinement protocol.

Third, the combination of the base map and a difference map is intended to represent a pure intermediate species. Therefore, alternative conformations in structural refinement that model a mixture of species would defeat this purpose. However, this combined map could be very noisy and may not represent a single species without a proper rotation. This is particular the case, if the target difference map $\Delta \rho$ is not derived from an SVD analysis and Ren rotation. The SVD analysis identifies many density components that are inconsistent among all observed difference maps and excludes them, which greatly reduces the noise content. Therefore, this refinement protocol may not be very successful without an SVD analysis. Another source of noise originates from the phase set of the structure factors. Prior to the refinement of the intermediate structure, the phase set remains identical to that of the ground state. This is far from the reality when an intermediate structure involves widespread changes, such as those refined in this study. If the rotation after SVD is not properly selected, the target difference map would remain as a mixture minus the ground state. Therefore, the refinement of a single conformation would encounter difficulty or significant residuals, as judged by the $R$ factors, the residual map, and the refined structure. A proper solution to this problem is a better SVD solution by Ren rotation rather than alternative conformations. A successful refinement of near perfect 


\section{Ren: Isomerization sampling}

993 trans or cis double bonds is a good sign to indicate that the reconstituted amplitude set 994 after a rotation reflects a relatively homogeneous structure. If a double bond could not 995 be refined well to near perfect trans or cis configuration, the dataset of structure factor 996 amplitudes is likely from a mixture of heterogeneous configurations, which occurred 997 frequently in previous studies of bR and photoactive yellow protein (Jung et al., 2013; 998 Lanyi and Schobert, 2007; Nogly et al., 2018). It has been a great difficulty in 999 crystallographic refinement in general that a heterogeneous mixture of conformations 1000 cannot be unambiguously refined even with alternative conformations. This difficulty 1001 becomes more severe when a mixture involves more than two conformations or when 1002 some conformations are very minor.

1003

Lastly, the refinement protocol proposed here could be carried out in the original 1005 unit cell and space group of the crystal at the ground state. However, this is not always 1006 applicable as the original goal of the meta-analysis is a joint examination of all available 1007 structures from a variety of crystal forms. It would be highly desirable to evaluate 1008 difference maps of the same or similar proteins from non-isomorphous crystals together 1009 by SVD. Alternatively, the refinement protocol could also be performed in the space 1010 group of P1 with a virtual unit cell large enough to hold the structure, which is the 1011 option in this study (Table S2). This is to say, the entire analysis of SVD-rotation1012 refinement presented here could be extracted and isolated from the original crystal 1013 lattices, which paves the way to future applications to structural data acquired by 1014 experimental techniques beyond crystallography, most attractively, to single particle 1015 reconstruction in cryo electron microscopy. 
Ren: Isomerization sampling

\section{Supplementary Tables}

Table S1. Datasets analyzed in this work

\begin{tabular}{|c|c|c|c|c|c|}
\hline Publication & PDB & Label & Resolution & Main conclusions & New findings in this work \\
\hline $\begin{array}{l}\text { Nogly et } \\
\text { al. Science } \\
361, \\
\text { eaat0094, } \\
2018\end{array}$ & $\begin{array}{l}6 \mathrm{~g} 7 \mathrm{~h} \\
6 \mathrm{~g} 7 \mathrm{i} \\
6 \mathrm{~g} 7 \mathrm{j} \\
6 \mathrm{~g} 7 \mathrm{k} \\
6 \mathrm{~g} 7 \mathrm{l}\end{array}$ & $\begin{array}{l}\text { dark6 } \\
49-406 \mathrm{fs} \\
457-646 \mathrm{fs} \\
10 \mathrm{ps} \\
8.33 \mathrm{~ms}\end{array}$ & $\begin{array}{l}1.5 \AA \\
1.9 \AA ̊ \\
1.9 \AA \\
1.9 \AA \\
1.9 \AA\end{array}$ & $\begin{array}{l}\text { Retinal fully } \\
\text { isomerizes by } 10 \\
\text { ps. But the SB } \\
\text { water dissociates } \\
\text { earlier. }\end{array}$ & $\begin{array}{l}\text { The short-delay datasets contribute to } \\
\text { the structures of } \mathrm{I}^{\prime} \rightarrow \mathrm{I} \rightarrow \mathrm{J}^{\prime} \rightarrow \mathrm{J} \text {. } \\
\text { Photoisomerization in } \mathrm{J}^{\prime} \text {; retinal } \\
\text { binding pocket expansion before } 1 \text { ps } \\
\text { in I and contraction at } 10 \text { ps in J }\end{array}$ \\
\hline $\begin{array}{l}\text { Kovacs et } \\
\text { al. Nat. } \\
\text { Commun. } \\
10,3177 \text {, } \\
2019\end{array}$ & $\begin{array}{l}\text { 6ga1 } \\
\text { 6ga2 } \\
\text { 6rmk } \\
6 \mathrm{ga7} \\
\text { 6ga8 } \\
\text { 6ga9 } \\
\text { 6gaa } \\
\text { 6gab } \\
\text { 6gac } \\
\text { 6gad } \\
\text { 6gae } \\
\text { 6gaf } \\
\text { 6gag } \\
\text { 6gah } \\
\text { 6gai } \\
\text { 6ga4 } \\
\text { 6ga5 } \\
\text { 6ga6 } \\
\text { 6ga3 }\end{array}$ & $\begin{array}{l}\text { dark1 } \\
\text { dark2 } \\
\text { dark3 } \\
240 \mathrm{fs} \\
330 \mathrm{fs} \\
390 \mathrm{fs} \\
430 \mathrm{fs} \\
460 \mathrm{fs} \\
490 \mathrm{fs} \\
530 \mathrm{fs} \\
560 \mathrm{fs} \\
590 \mathrm{fs} \\
630 \mathrm{fs} \\
680 \mathrm{fs} \\
740 \mathrm{fs} \\
1 \mathrm{ps} \\
3 \mathrm{ps} \\
10 \mathrm{ps} \\
33 \mathrm{~ms}\end{array}$ & 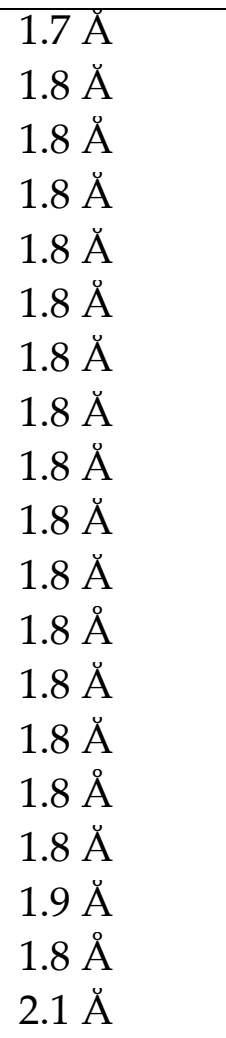 & $\begin{array}{l}\text { The exceedingly } \\
\text { high power } \\
\text { density of the } \\
\text { pump laser } \\
\text { causes two- } \\
\text { photon } \\
\text { absorption. } \\
\text { Vibrational } \\
\text { motions were } \\
\text { observed. }\end{array}$ & $\begin{array}{l}\text { The sub-ps datasets exhibit extensive } \\
\text { vibrations at various frequencies. } \\
\text { The vibrational signals are } \\
\text { widespread over the entire bR } \\
\text { molecule and not associated with any } \\
\text { structural elements. Therefore, it is } \\
\text { concluded that these global } \\
\text { vibrations are intrinsic properties of } \\
\text { bR induced by short laser pulses. } \\
\text { The vibrational signals are more } \\
\text { prominent under higher power } \\
\text { density of the laser pulses. However, } \\
\text { these vibrations are irrelevant to the } \\
\text { light-driven proton pumping } \\
\text { function of bR. }\end{array}$ \\
\hline
\end{tabular}

1019

1020 
Ren: Isomerization sampling

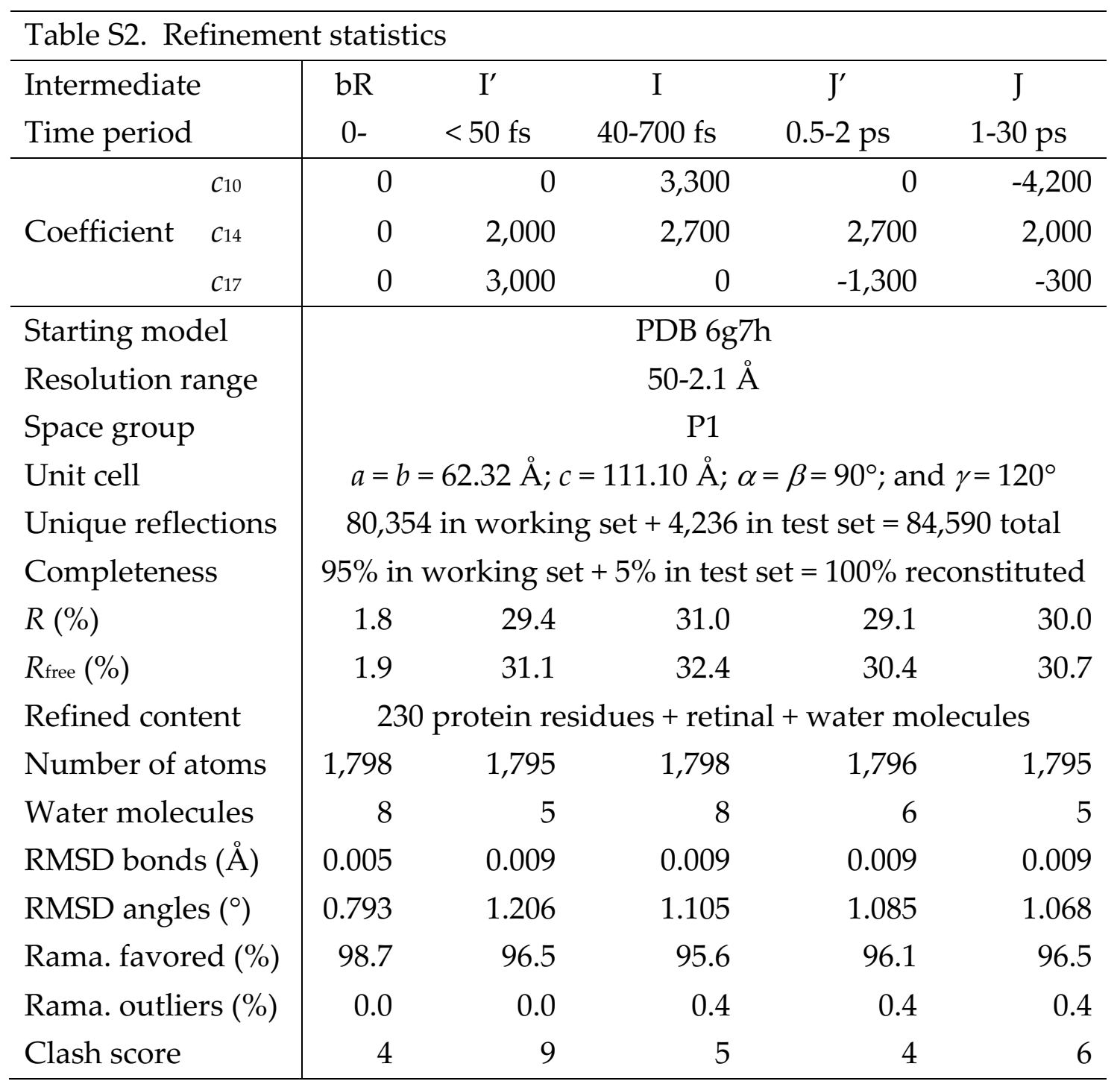


1023 Supplementary Figures and Legends

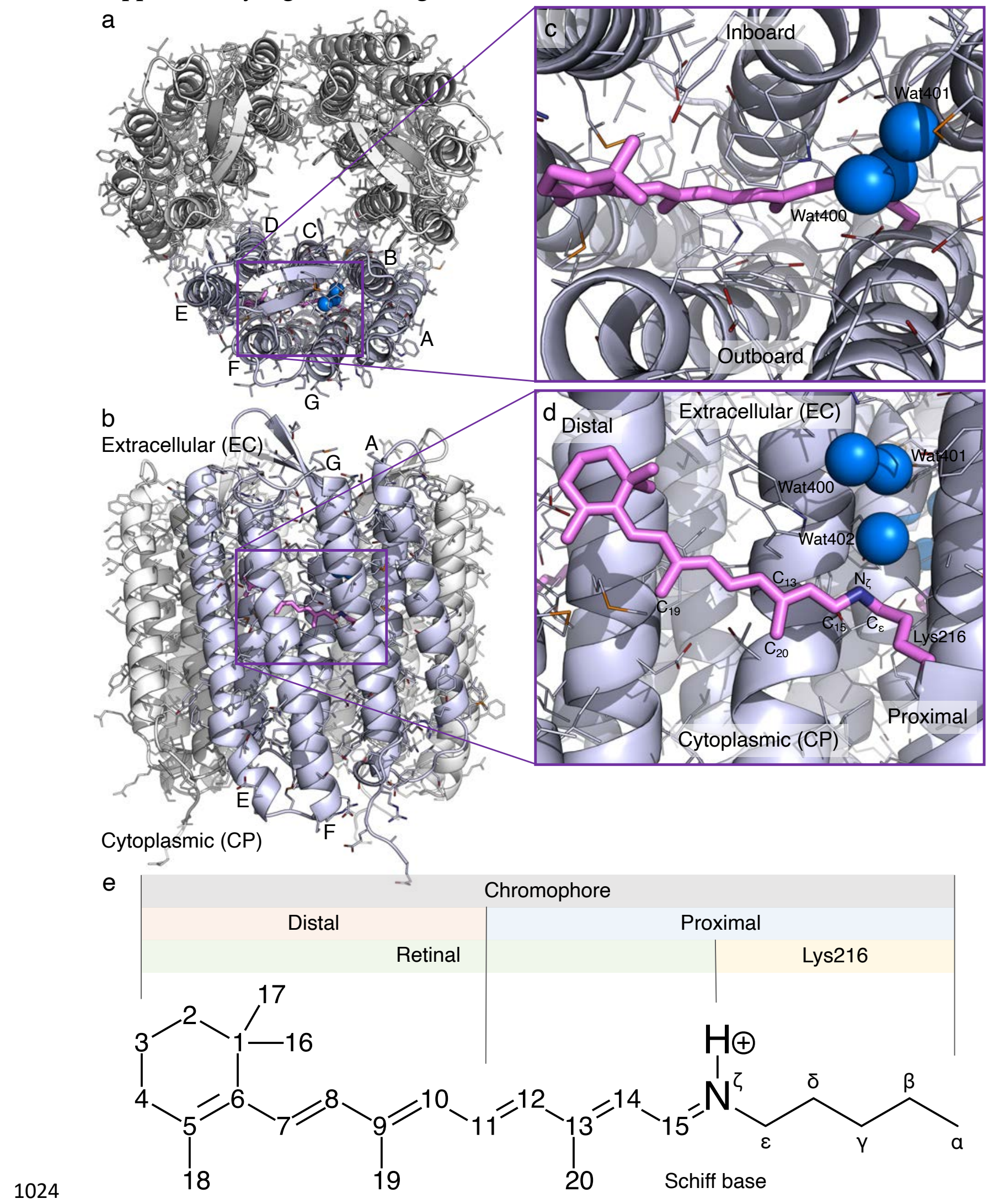




\section{Ren: Isomerization sampling}

1025 Figure S1. Orientations in bacteriorhodopsin. (a) Bacteriorhodopsin (bR) trimer viewed 1026 from the extracellular (EC) side along the three-fold axis. (b) An orthographical view to 1027 (a) looking from the outside of the trimer. (c and d) Two orthographical views of the 1028 retinal chromophore looking along the three-fold and normal to the three-fold axis. The 1029 plane of retinal is largely parallel to the three-fold axis. Therefore, two sides of the 1030 plane are called inboard and outboard with respect to the three-fold axis. The direction 1031 toward the anchor Lys216 is called proximal. The $\beta$-ionone ring direction is therefore 1032 distal. (e) Chemical structure of retinal incorporated to its anchor Lys216. The atom 1033 numbers and various segment names are marked. 
Ren: Isomerization sampling
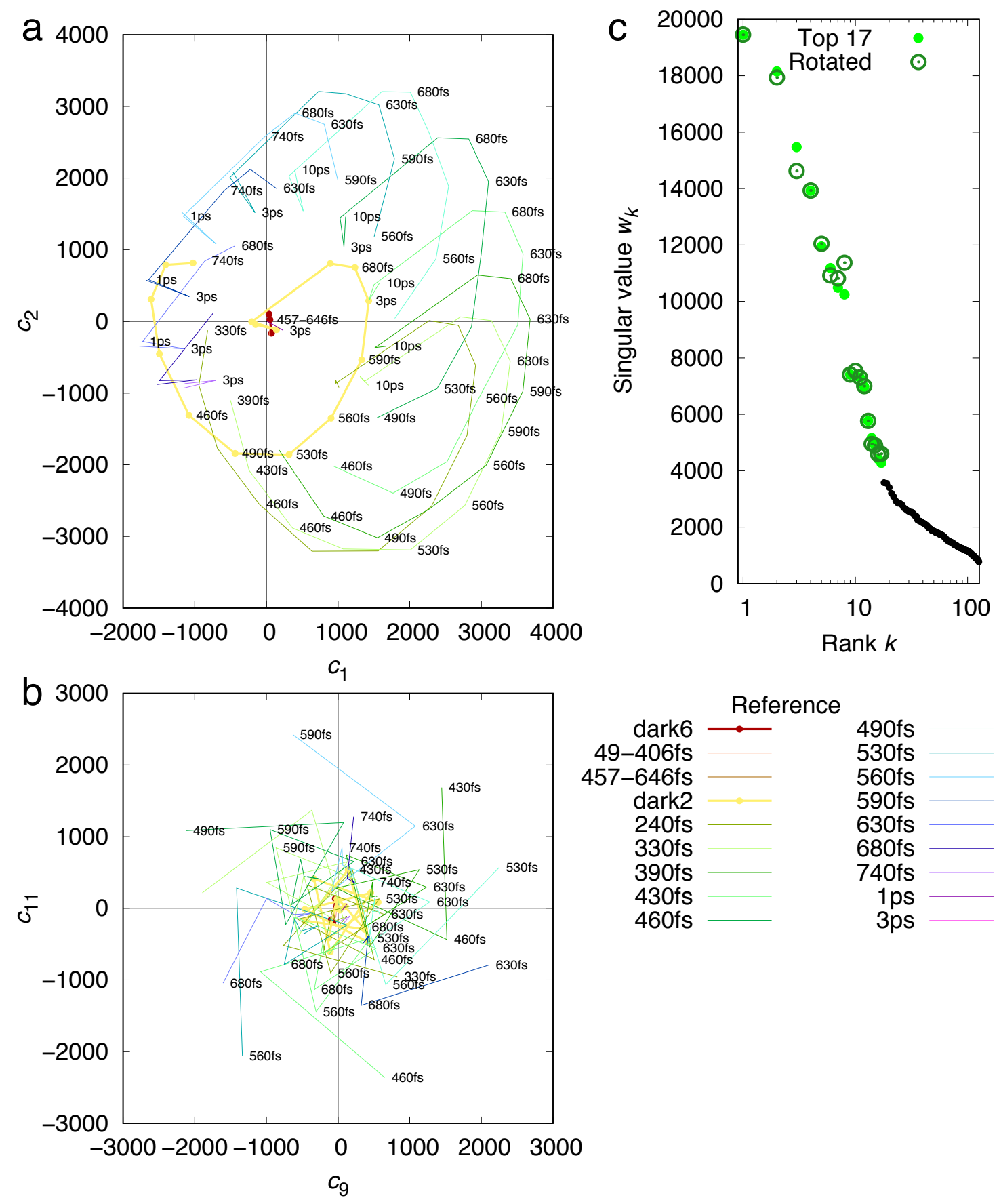

1037 Figure S2. SVD applied to difference Fourier maps. Difference Fourier maps at the 1038 short delays $t \leq 10$ ps are decomposed into component maps. Each difference map at a 1039 time delay $t$ can be represented by a linear combination of these components, $c_{1}(t) \boldsymbol{U}_{1}+$ $1040 c_{2}(t) \boldsymbol{U}_{2}+\ldots$, where $\boldsymbol{U}_{k}$ are the time-independent components and $c_{k}(t)$ are their 1041 corresponding time-dependent coefficients (Methods). (a and b) Two example plots 1042 show circular correlations between $c_{1}$ and $c_{2}, c_{9}$ and $c_{11}$. These circular correlations 
Ren: Isomerization sampling

1043 indicate two-dimensional oscillations. Each colored trace represents difference maps in 1044 a time series calculated with a common reference. Those time series with a dark 1045 reference are plotted with thick lines. Other series are in thin lines. (c) Singular values 1046 before and after Ren rotation (Ren, 2016, 2019) (Methods). Singular values derived from 1047 SVD indicate the significance of the components. 17 of them stand out. 
Ren: Isomerization sampling
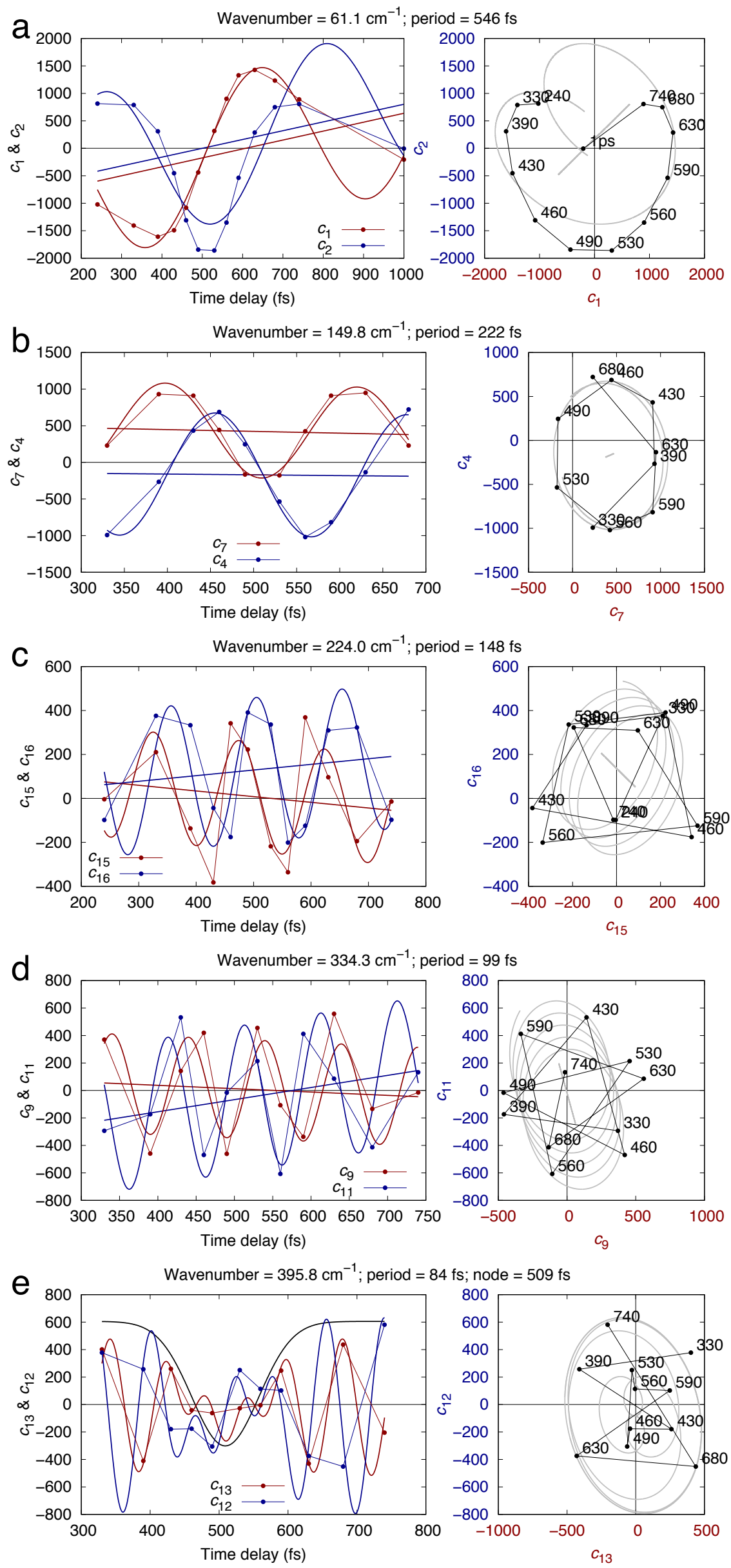


\section{Ren: Isomerization sampling}

1050 Figure S3. Oscillations of SVD components. The coefficients of ten components $c_{1}, c_{2} ; c_{4}$, $1051 C C_{7} ; C_{15}, C_{16} ; C_{9}, C_{11}$; and $c_{12}, c_{13}$ are found oscillating at frequencies ranging from 60 to 400 $1052 \mathrm{~cm}^{-1}$. Each pair of the coefficients oscillate at a common frequency. These frequencies 1053 are $61 \pm 2,150 \pm 3,224 \pm 7,334 \pm 8$, and $396 \pm 3 \mathrm{~cm}^{-1}$, respectively. These coefficients are 1054 plotted against the time delay $t$ (left) and against each other in a pair (right). Each 1055 coefficient is fitted with a sine function around a straight baseline $c_{k}=a \sin \left(\frac{2 \pi t}{T}+\varphi\right)+$ $1056 b+c t$. Both the fitted function and the baseline are plotted. The amplitude $a$ for the 1057 last pair of coefficients $c_{12}$ and $c_{13}$ are replaced with a Gaussian function $a-$ $1058 \exp \left(-\frac{\left(t-t_{0}\right)^{2}}{\tau^{2}}\right)$ to implement a node at $t_{0}=509 \pm 5 \mathrm{fs}(\mathrm{e})$.

1059

1060 


\section{Ren: Isomerization sampling}
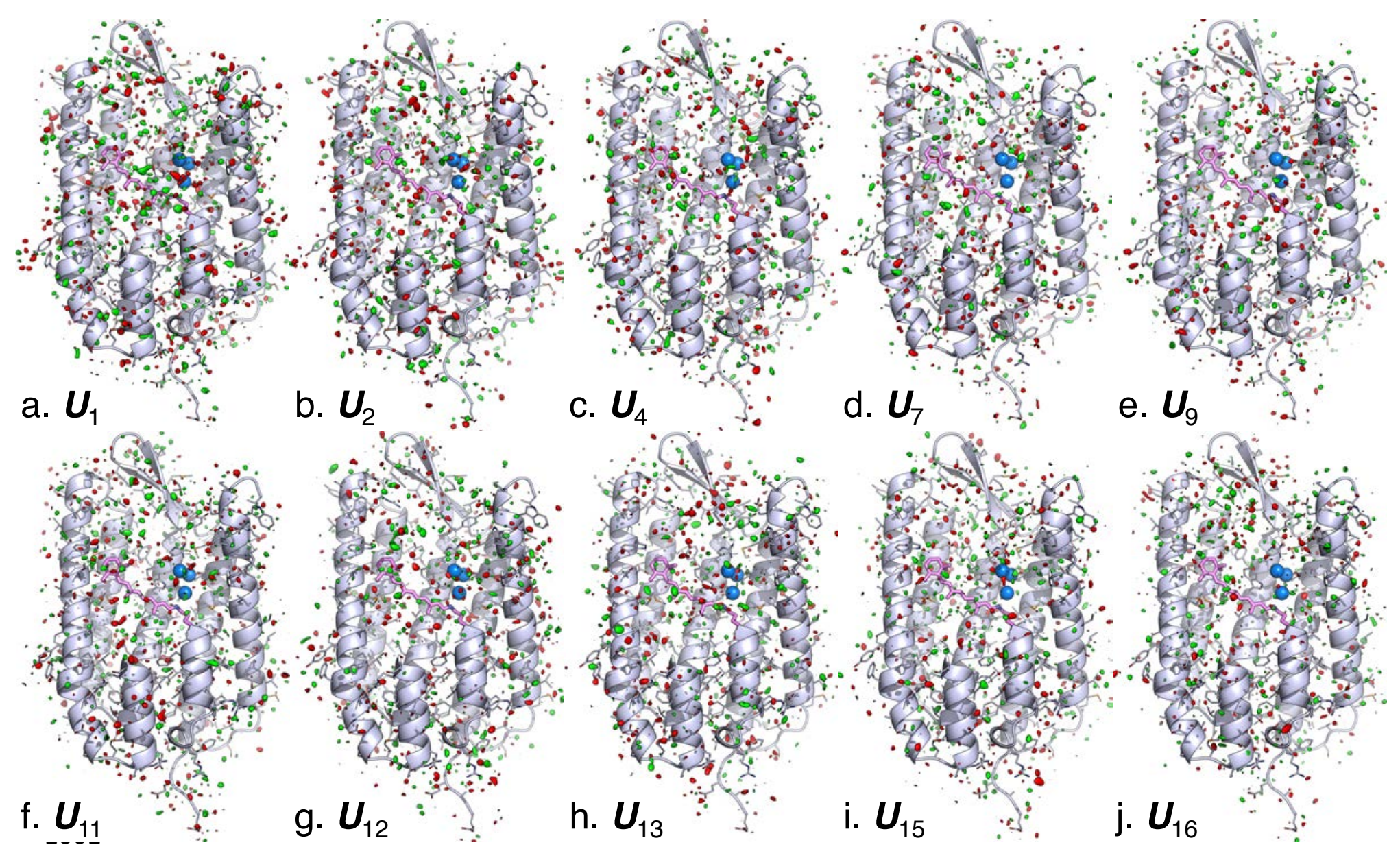

1062

1063 Figure S4. No structural signal in oscillating components. Ten oscillating components

1064 are contoured at $\pm 3 \sigma$ in green and red, respectively. The main chain and side chains of

1065 the protein are rendered with ribbon and sticks, respectively. The retinal and Lys216

1066 are in purple sticks. Several key waters are in blue spheres. Parts of the structure are

1067 omitted to reveal more of the interior. Despite that the time-dependent coefficients to

1068 these components contain strong oscillatory signals (Figs. S2 and S3), these components

1069 themselves display no obvious association with any structural features such as the

1070 retinal or secondary structures. They are in stark contrast to the signal distributions of

1071 the non-oscillating components (Figs. 1ab, 2b, and S5).

1072 
Ren: Isomerization sampling

1073

1074

1075

1076

1077

1078

1079

1080

1081
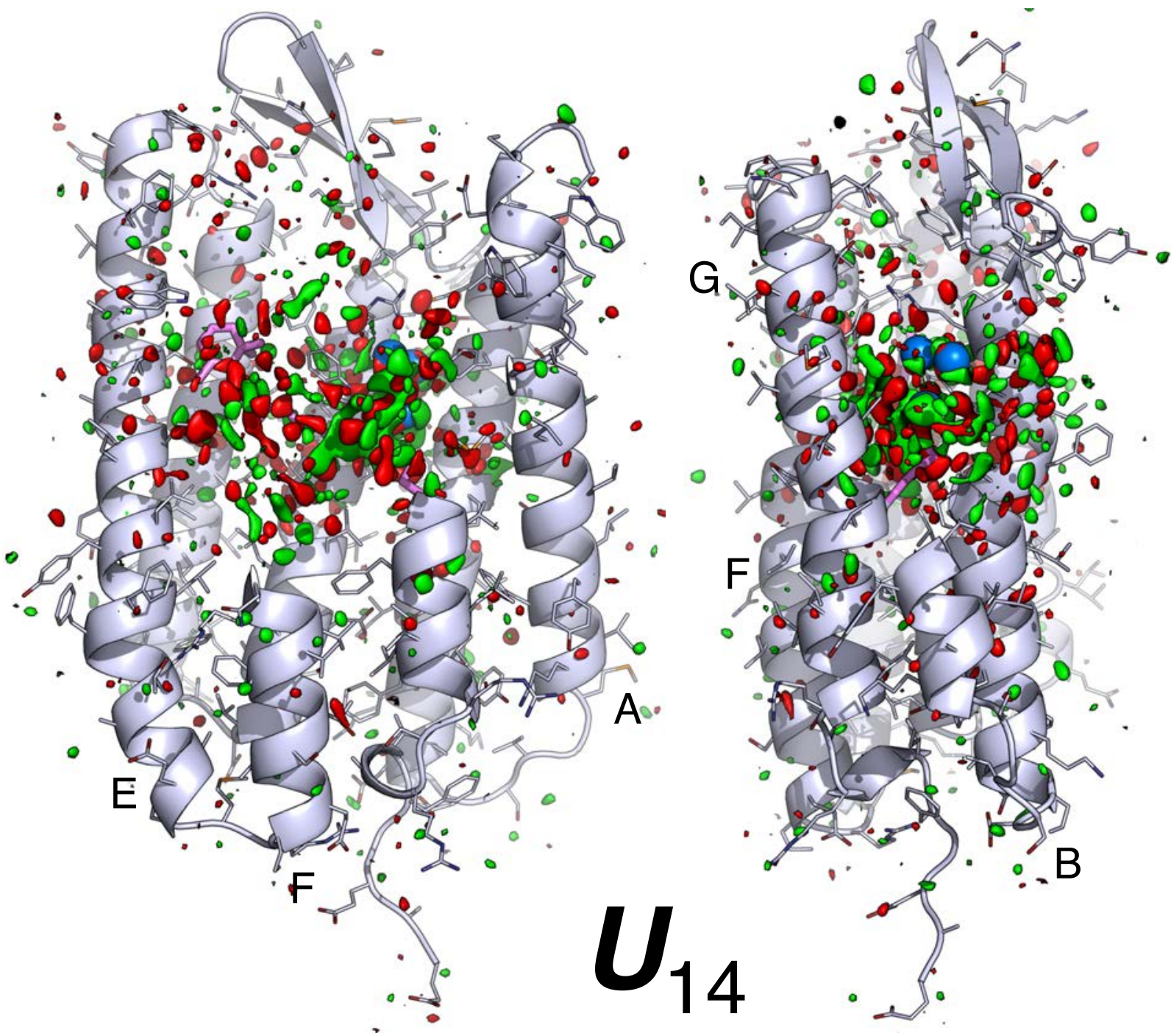

Figure S5. Two orthographical views of component map $\boldsymbol{U}_{14}$. The main chain and side chains of the protein are rendered with ribbons and sticks, respectively. The retinal and Lys216 are in purple sticks. Several key waters are in blue spheres. Parts of the structure are omitted to reveal more of the interior. The map is contoured at $\pm 3 \sigma$ in green and red, respectively. The signals are largely associated with the chromophore and its immediate vicinity. 
Ren: Isomerization sampling

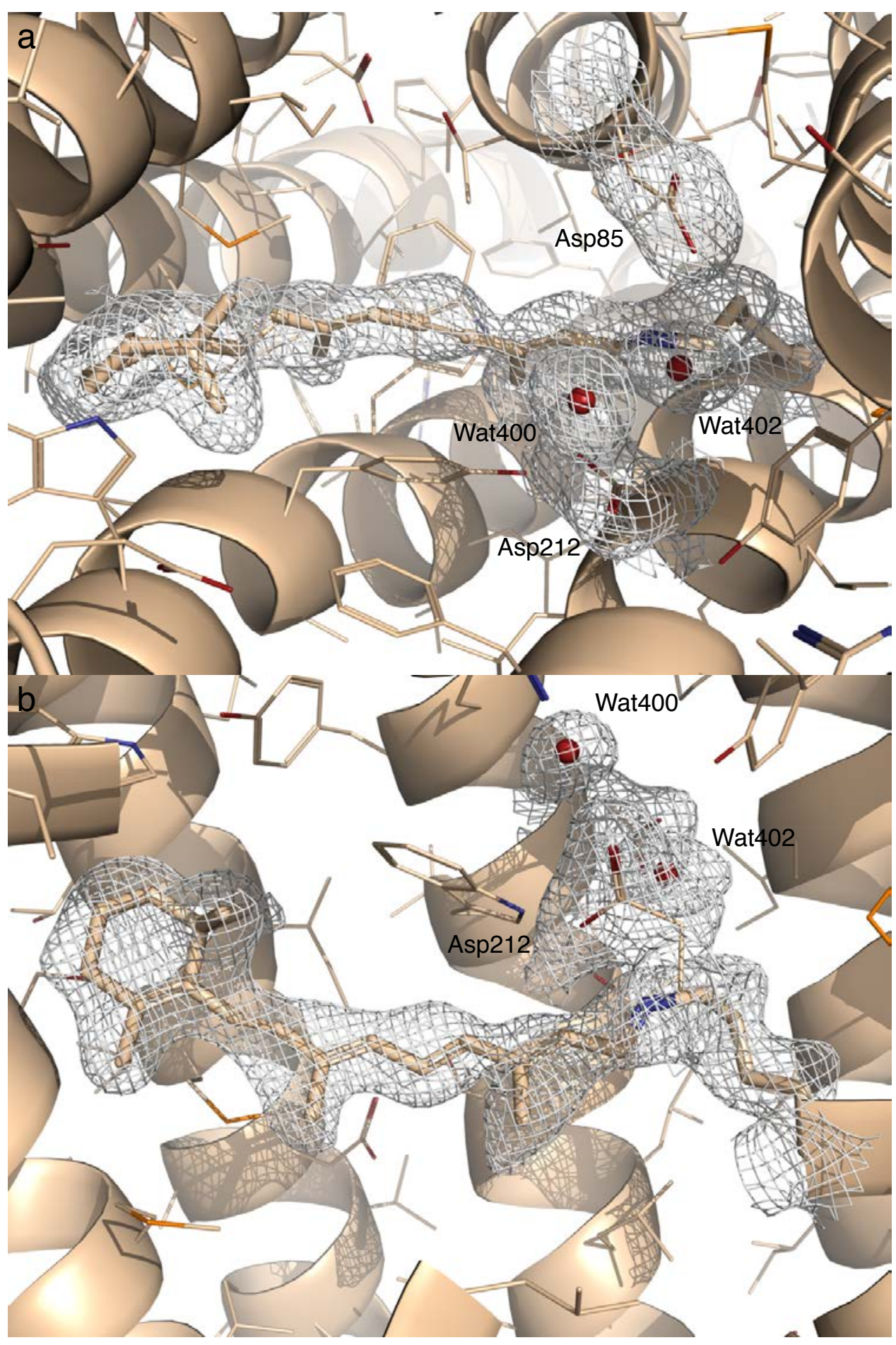

1084 Figure S6. Two orthographical views of the 2Fo-Fc map of I' contoured at $3.5 \sigma$. Here Fo 1085 is the reconstituted structure factor amplitudes rather than observed amplitudes (Table 1086 S2). Fc is the structure factor amplitudes calculated from the refine structure (Methods). 
bioRxiv preprint doi: https://doi.org/10.1101/2021.09.16.460656; this version posted September 16, 2021. The copyright holder for this preprint (which was not certified by peer review) is the author/funder. All rights reserved. No reuse allowed without permission.

Ren: Isomerization sampling

1087 The same protocol applies to the Fourier synthesis of 2Fo-Fc maps of other

1088 intermediates (Figs. S7, S9, and S10).

1089

1090 
Ren: Isomerization sampling

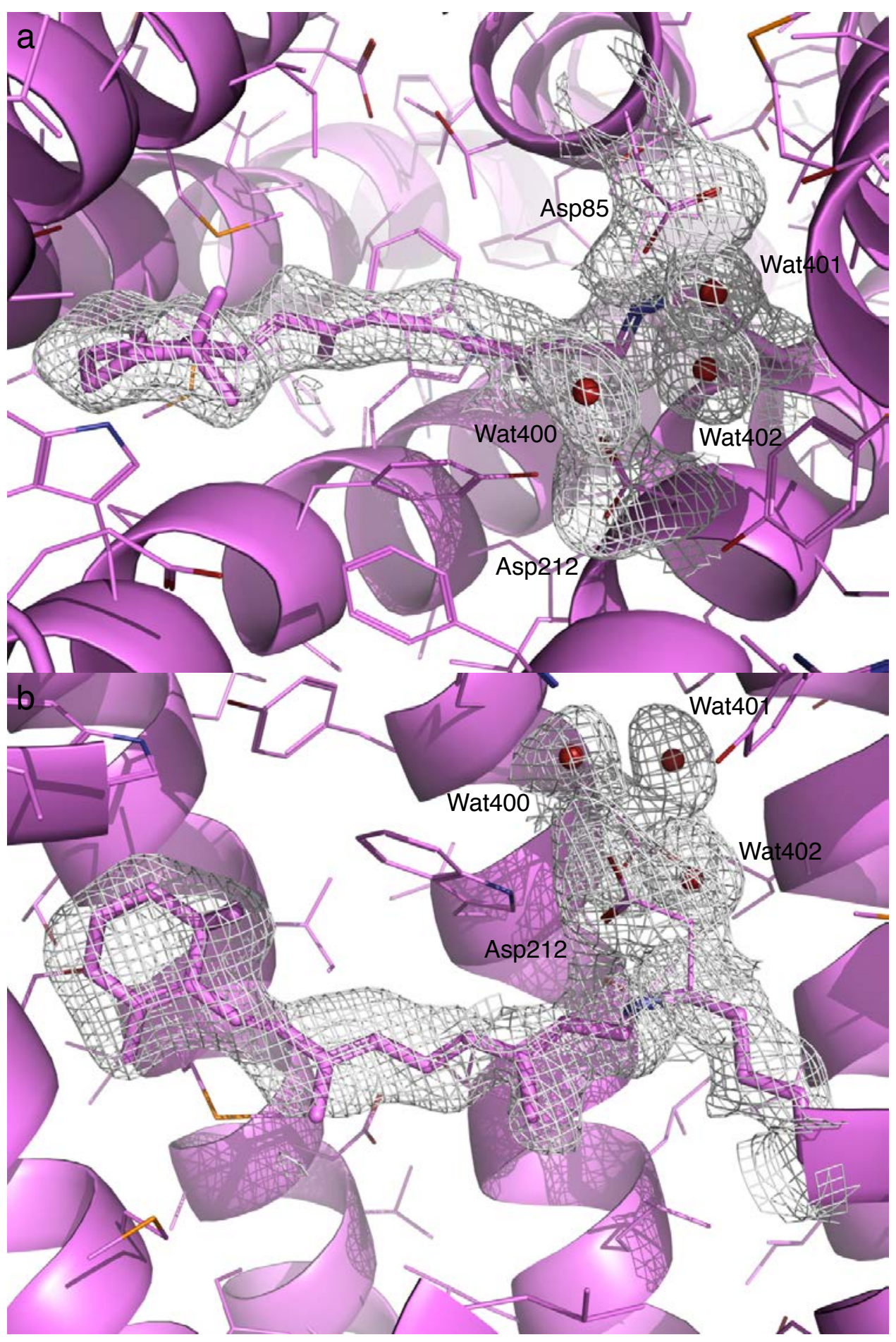

1093 Figure S7. Two orthographical views of the 2Fo-Fc map of I contoured at $3 \sigma$. Here Fo is 1094 the reconstituted structure factor amplitudes rather than observed amplitudes (Table 1095 S2). Fc is the structure factor amplitudes calculated from the refine structure (Methods). 
Ren: Isomerization sampling

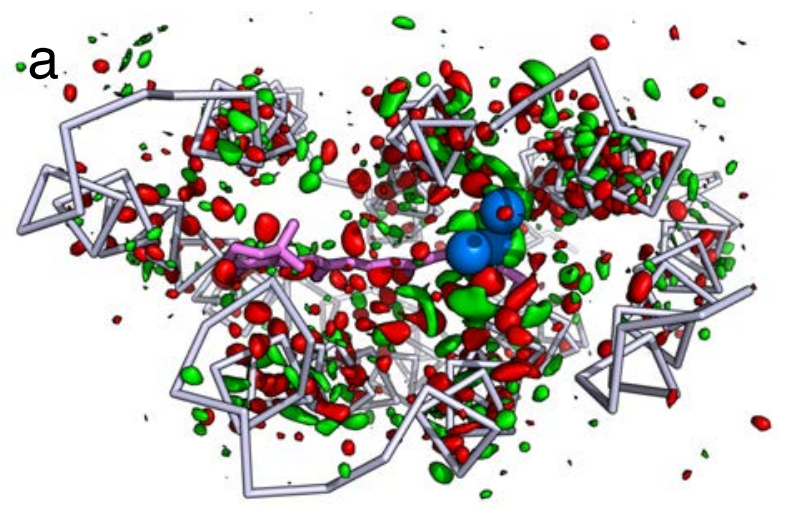

$6 g 7 j-6 g 7 h$
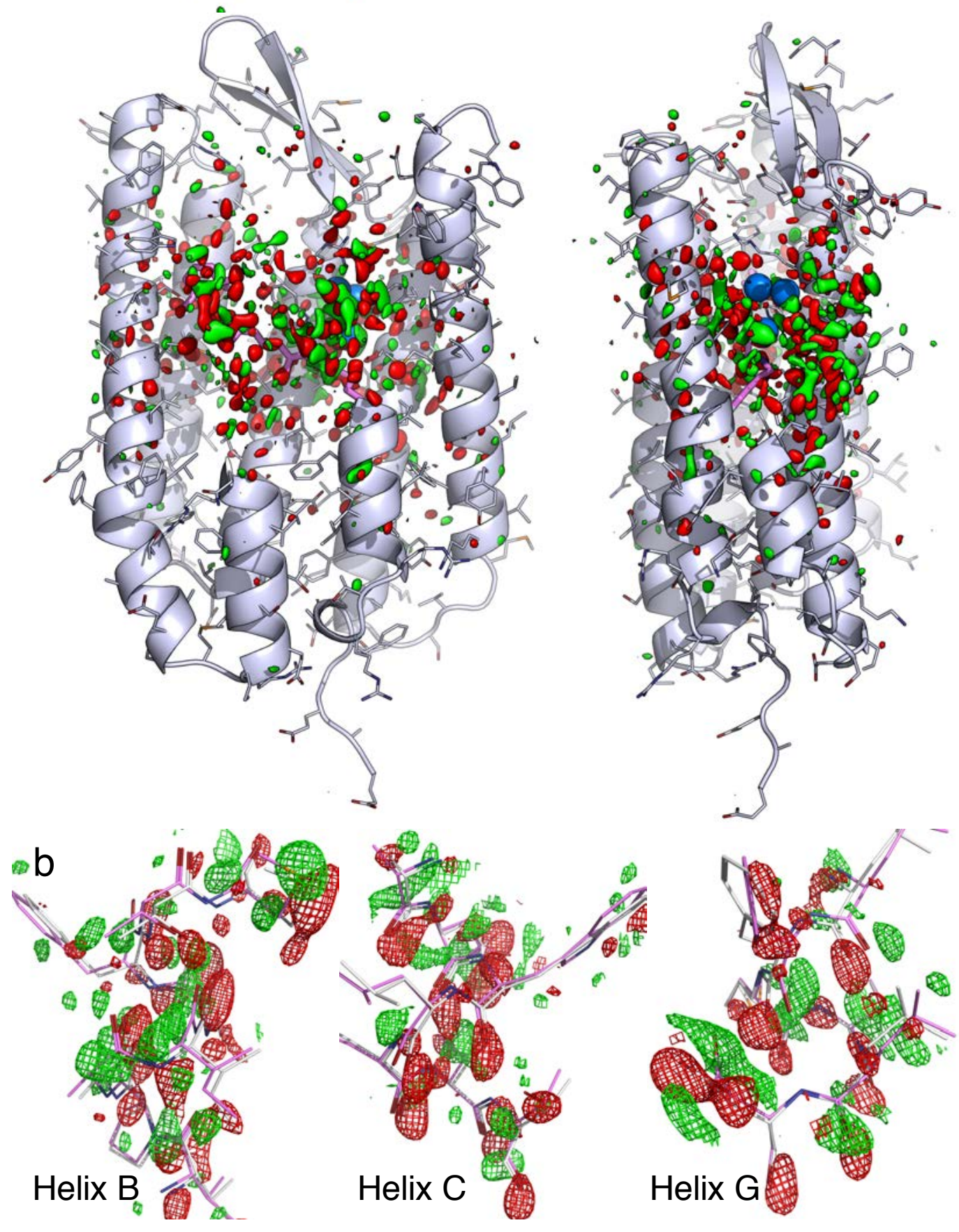


\section{Ren: Isomerization sampling}

1098 Figure S8. Raw difference Fourier map at 457-646 fs. This difference Fourier map is 1099 calculated from the dataset 6g7j at the time point of 457-646 fs by subtracting the dark 1100 dataset $6 \mathrm{~g} 7 \mathrm{~h}$. The map is contoured at $\pm 3 \sigma$ in green and red, respectively. This map is 1101 prior to SVD analysis. Compared with $\boldsymbol{U}_{10}$ (Fig. 2b) and the reconstituted map (Fig. 3a), 1102 it is clear that this is the original source of the widespread signals except that the $\sigma$ 1103 value of this map is higher than those after SVD. (a) The raw difference map contoured 1104 in the entire molecule shows the association of the signals with the structural elements 1105 at an excellent signal-to-noise ratio. (b) Details of the raw difference map show 1106 displacements of helices. The raw difference map is largely the same as the 1107 reconstituted map (Fig. 3a). 
Ren: Isomerization sampling

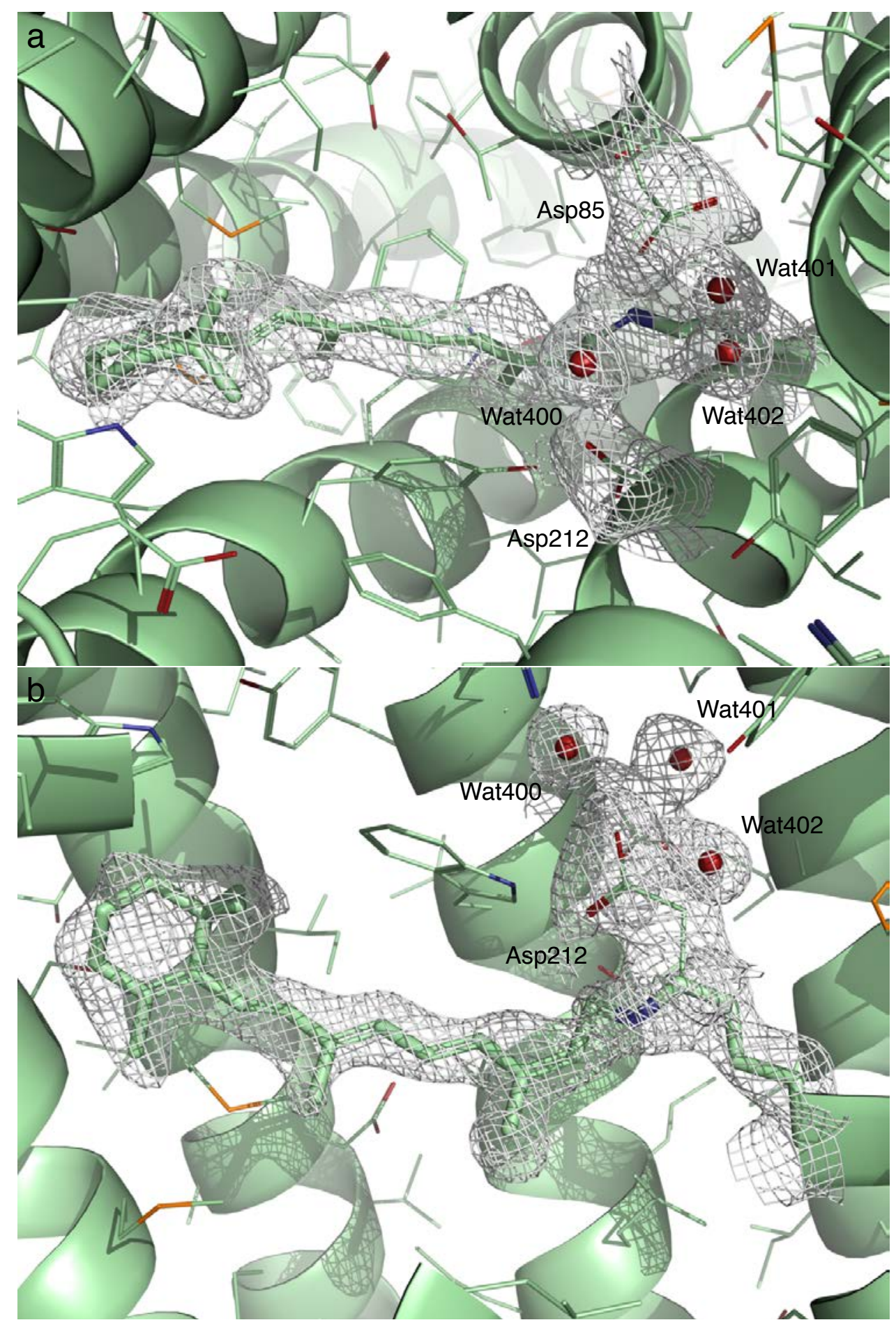

1112 Figure S9. Two orthographical views of the 2Fo-Fc map of J' contoured at $4 \sigma$. Here Fo 1113 is the reconstituted structure factor amplitudes rather than observed amplitudes (Table 1114 S2). Fc is the structure factor amplitudes calculated from the refine structure (Methods). 
Ren: Isomerization sampling

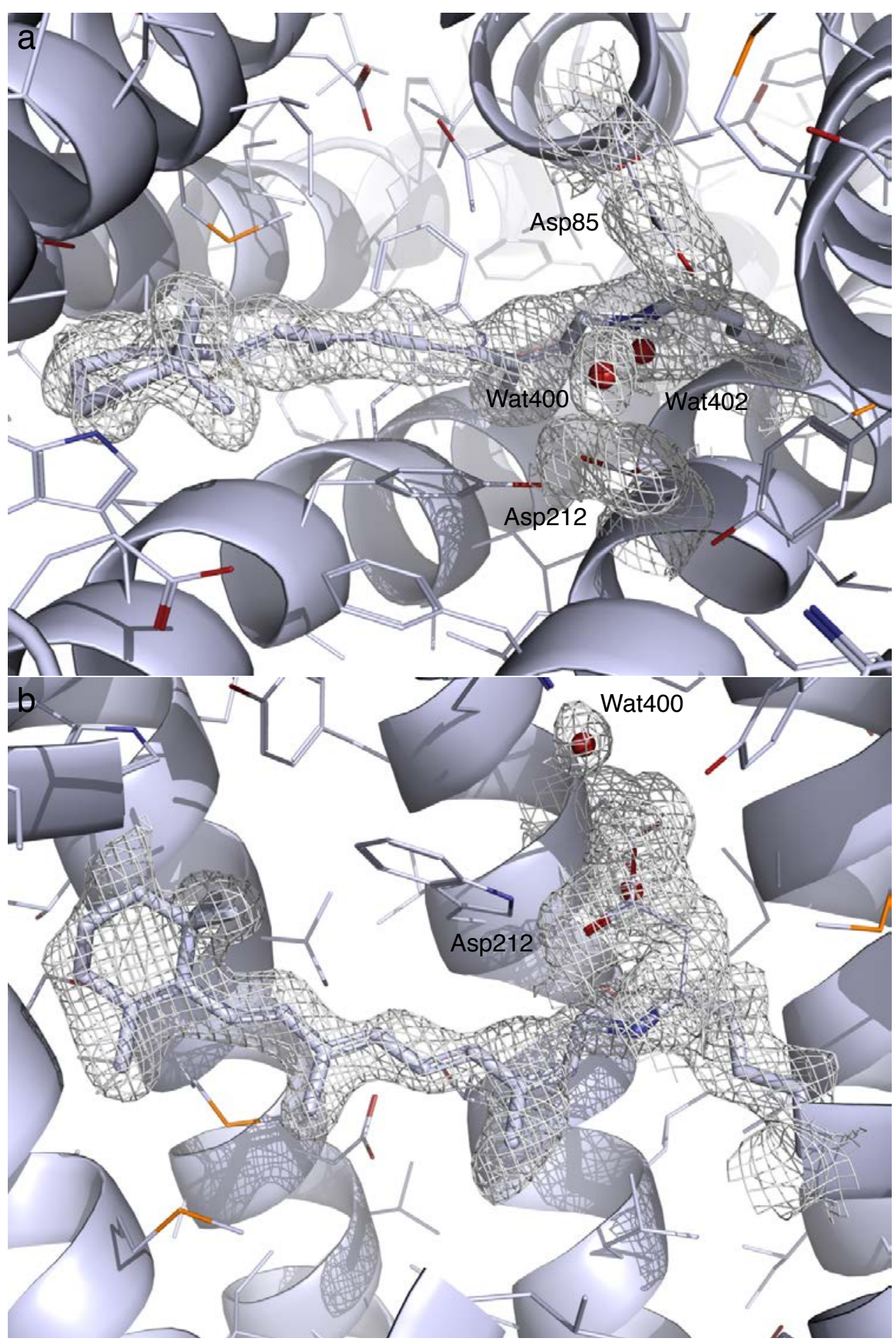

1118 Figure S10. Two orthographical views of the $2 \mathrm{Fo}-\mathrm{Fc}$ map of J contoured at $5 \sigma$. Here Fo 1119 is the reconstituted structure factor amplitudes rather than observed amplitudes (Table 1120 S2). Fc is the structure factor amplitudes calculated from the refine structure (Methods). 